\title{
ORIBÁTIDOS (ACARI, ORIBATEI) DE FUERTEVENTURA (ISLAS CANARIAS)
}

\author{
C. Pérez-Iñigo (*) y M.A. Peña $(* *)$
}

\begin{abstract}
RESUMEN
Se estudian por primera vez los ácaros oribátidos de Fuerteventura, y se citan 86 especies de las que 18 son nuevas para la Ciencia y dos son nuevas subespecies, y se establecen dos géneros nuevos: Passalobates (Familia Passalozetidae) y Fuerteventuria (Familia Protoribatidae). Se describen dos especies de géneros etiópicos: Scutoverticosus insperatus n. sp. y Ethiovertex elisae n. sp., géneros que, hasta ahora, solo contaban con la especie tipo.
\end{abstract}

Palabras clave: Acari, Oribatei, Canarias, Fuerteventura

\section{ABSTRACT}

\section{Oribatid mites (Acari, Oribatei) from Fuerteventura (Canary Islands)}

The oribatid fauna from Fuerteventura (Canary Islands) is studied for the first time; 86 species are recorded, from which 18 are new to the Science and two are new subspecies. Two new genera are erected: Passalobates (Family: Passalozetidae) and Fuerteventuria (Family Protoribatidae). Two species belonging to Ethiopic genera are described: Scutoverticosus insperatus $\mathrm{n}$. sp. and Ethioverthex elisae n. sp. These genera contained until now the type species only.

Key words: Acari, Oribatei, Canary Islands, Fuerteventura

\section{Introducción}

El presente trabajo es el primero sobre los ácaros oribátidos de Fuerteventura. Se han estudiado 82 muestras de suelo, de las que 65 contenían oribátidos identificables; dichas muestras fueron obtenidas por el segundo de los autores, excepto un corto número que lo fue por el señor S. Scholz, y han arrojado un total de 3.084 ejemplares, pertenecientes a 86 especies, distribuídas en 47 familias. Se han encontrado 18 especies y 2 subespecies nuevas para la Ciencia y se han creado dos nuevos géneros.

\section{Material y Métodos}

Lista de Muestras (en cada localidad se indican los metros sobre el nivel del mar y las coordenadas U.T.M.)

F-1. Montaña de los Verodes, 17-VI-94. Mantillo de Ononix natrix $140 \mathrm{~m}$., 28RES743132.

F-2. Idem, idem. suelo de caliche entre raices de Ononix natrix, $120 \mathrm{~m}$., 28RES743133.

F-3. Degollada de los Verodes, 17-VI-94. Interior desmenuzado de tronco de Phoenix dactylifera, $100 \mathrm{~m}$. 28RES743134

F-5. Barranco Tras el Lomo, 17-VI-94. Suelo y mantillo de Salsola marujae y Euphorbia regis-jubae, $140 \mathrm{~m}$ 28RES 824291

\footnotetext{
* Museo Nacional de Ciencias Naturales, calle de José Gutiérrez Abascal, 2. 28006 MADRID

** Calle Ocho de Marzo,1. Edificio Cantoneras, 5, 3 A. 35200 TELDE (Las Palmas, Islas Canarias)
} 
F-6. Degollada del Barranco Los Canarios, 17-VI-94. Líquenes sobre piedras: Ramalina bourgeana y Rochella sp., 320 m. 28RES662111

F-7. Pico del Viento, 17-VI-94. Suelo y mantillo de Klenia nerifolia y Lycium intricatum, 80 m. 28RES659112

F-8. Valle de los Mosquitos, 18-VI-94. Suelo y restos secos de Euphorbia handiensis, Suaeda vera y Lycium intricatum, 80 m. 28RES569055

F-9. Rabo del Ratón, 18-VI-94. Suelo y mantillo seco de Zygophyllum gaetulum, expuesto al spray marino, $15 \mathrm{~m}$. 28RES505092

F-10. Casas de Cofete, 18-VI-94. Suelo con Euphorbia canariensis, $100 \mathrm{~m}$. 28RES584082

F-14. Barranco Vinamar, 23-VII-94. Suelo y mantillo de Onopordon nogalesii y Klenia nerifolia, $250 \mathrm{~m}$. 28RES647065

F-15. Bco. Vinamar, 23-VII-94. Líquenes sobre pared basáltica con Ramalina bourgeana, Rochella canariensis, $R$. tuberculata, etc., $380 \mathrm{~m}$. 28RES638077

F-16 Bco. Vinamar, 23-VII-94. Suelo con musgos secos entre raíces de Euphorbia canariensis, $380 \mathrm{~m}$. 28RES638077

F-17. Saladar el Matorral, 23-VII-94. Suelo arenoso y mantillo de Arthrocnemum fruticosum, Frankenia laevis y Suaeda vera, $2 \mathrm{~m}$. 28RES672034

F-18. Montaña Pelada, 24-VII-94. Suelo arenoso y mantillo de Launaea arborescens y Salsola marujae, $85 \mathrm{~m}$. 28RES725125

F-19. Malpaís de Pozo Negro, 24-VII-94. Suelo arcilloso y mantillo de Salsola vermiculata en campo de lavas, 50 m. 28RFS057341

F-21. Malpaís de Huriame, 24-VII-94. Sobre piedra basáltica con Xanthoria resendei, 90 m. 28RFS085742

F-22. Roca La Mar (El Cotillo), 24-VII-94. Suelo y mantillo de Zygophyllum fontanesii y Pulicaria canariensis, 10 m. 28RES9666729

F-24. Barranco Los Molinos, 24-VII-94. Suelo y mantillo de matorral denso de Suaeda vera, 10 m. 28RES919575

F-25. Tabla de Vinamar, 25-VII-94. Suelo y mantillo de Euphorbia regis-jubae y Lycium intricatum, $500 \mathrm{~m}$. 28RES648071

F-26. Pico de La Zarza, 25-VII-94. Suelo húmedo y mantillo de Nauplius sericeus, 780 m. 28RES635084

F-27. Pico de La Zarza, 25-VII-94. Suelo húmedo con musgos, bajo Nauplius sericeus, 800 m. 28RES635085

F-28. Pico de La Zarza, 25-VII-94. Suelo muy húmedo de 3 $\mathrm{cm}$ de profundidad, con musgos. $807 \mathrm{~m}$. 28RES655085

F-29. Pico de La Zarza, 25-VII-94. Suelo de pared norte muy húmeda, con líquenes diversos. 790 m. 28RES636086

F-30. Pico de La Zarza, 25-VII-94. Suelo con lapilli en cueva de pared norte muy húmeda, con gramínea cespitosa, 780 m. 28RES636086

F-31. Pico de La Zarza, 25-VII-94. Hojas, corteza y troncos muertos de Nauplius sericeus, 780 m. 28RES636086

F-32. Barranco de Butihondo, 25-VII-94. Suelo seco de Euphorbia canariensis, 150 m. 28RES663068

F-33. Tesejeraque, 26-VII-94. Suelo muy seco y mantillo de Salsola vermiculata y Launaea arborescens, $150 \mathrm{~m}$. 28RES894281

F-34. Lomo del Aceituno, 26-VII-94. Suelo seco y mantillo de Euphorbia balsamifera y Salsola vermiculata, $340 \mathrm{~m}$. 28RES882384
F-35. Barranco Betancuria, 26-VII-94. Suelo y mantillo seco de Tamarix canariensis, 3 m. 28RES919421

F-37. Barranco de Las Damas, 27-VII-94. Suelo húmedo todo el año, junto rezumadero con Apium nodiflorum y Adiantum capillus-veneris, 250 m. 28RES605069

F-38. Barranco de Las Damas, 27-VII-94. Suelo y mantillo bajo Euphorbia balsamifera en zona con alta humedad atmosférica, 250 m. 28RES605069

F-39. Esquinzo-Jandía, 4-II-94. Tierra húmeda de jardín, 50 m (S. Scholz leg.). 28RES690059

F-40. Esquinzo-Jandía, 4-II-94. Compost de jardín, 50 m. (S. Scholz leg.). 28RES690059

F-41. Malpaís Pozo Negro, 4-II-94. Tierra seca con algo de mantillo, $50 \mathrm{~m}$ (S. Scholz leg.). 28RFS057341

F-43. Barranco de La Torre, 12-XII-94. Suelo arenoso y mantillo de Tamarix canariensis, $55 \mathrm{~m}$. 28RFS070390

F-44. Corral Bermejo, 13-XII-94. Suelo muy seco y encalichado con mantillo de Lycium afrum, $390 \mathrm{~m}$. 28RES641047

F-46. Casas de Jorós, 13-XII-94. Suelo arenoso y restos secos de raíces de Euphorbia canariensis, $100 \mathrm{~m}$. 28RES584082

F-47. Degollada del Bco. Los Canarios, 14-XII-94. Líquenes sobre piedras y troncos secos de Nauplius sericeus, 360 m. 28RES657108

F-48. Degollada del Bco. Los Canarios, 14-XII-94. Suelo entre raíces de Launaea arborescens y bajo piedras, $360 \mathrm{~m}$. 28RES655109

F-49. Tablero de Betancores, 14-XII-94. Suelo arenoso al pie de Suaeda sp., 50 m. 28RES530065

F-51. Puerto Lajas, 1-IV-95. Arena organógena y mantillo de Zygophyllum fontanesii en línea supramareal, $0 \mathrm{~m}$. 28RFS139569

F-52. Malpaís de Roja, 1-IV-95. Suelo de malpaís muy seco con mantillo de Suaeda vera, $52 \mathrm{~m}$. 28RFS138671

F-54. Majanicho, 1-IV-95. Suelo arcilloso en depresión dentro del malpaís a $50 \mathrm{~m}$ de línea de marea y con mantillo de Suaeda vera, 5m. 28RFS034795

F-55. Las Vistas, 1-IV-95. Líquenes foliáceos sobre piedras secas del malpaís, $50 \mathrm{~m}$. 28RFS039768

F-58. Montaña Tindaya, ladera sur, 1-IV-95. Suelo y mantillo de gramíneas, Opuntia dilemii y Lycium intricatum, 200 m. 28RES999631

F-59. Vallebrón, 1-IV-95. Suelo arenoso y mantillo de Nauplius sericeus en el cauce del barranco, $270 \mathrm{~m}$. 28RFS038614

F-60. Vallebrón, I-IV-95. Suelo arenoso y mantillo de Ficus carica en el cauce del barranco, 270 m. 28RFS038614

F-61. Montaña La Muda, 1-IV-95. Suelo mineral bruto y mantillo de Lycium intricatum y terófitos, al pie de un muro de piedra seca, $500 \mathrm{~m}$. 28RFS023604

F-62. Montaña La Muda, pista en ladera norte, 1-IV-95. Líquenes foliáceos sobre pared basáltica, $400 \mathrm{~m}$. 28RFS027602

F-64. Llanos de la Concepción, 1-IV-95. Tierra arcillosa en llanura central y árida con Suaeda vera, $180 \mathrm{~m}$. 28RES952504

F-65. Morro de Velosa, ladera oeste, 1-IV-95. Suelo y mantillo de Euphorbia balsamifera, Lotus lacerottensis y Pinus sp, 600 m. 28RES988459

F-66. El Fuerte (cerca de Antigua), 1-IV-95. Tierra y mantillo en suelo arcilloso con gramíneas y Launaea arborescens, 290 m. 28RES968454 
F-67. Agua de Bueyes, 1-IV-95. Tierra y mantillo de Ceratonia siliqua, $250 \mathrm{~m}$. 28RES953382

F-68. Tiscamanita, 1-IV-95. Tierra y mantillo entre raíces de Phoenix dactylifera al borde de una "gavia", $230 \mathrm{~m}$. 28RES943364

F-69. Tuineje, 1-IV-95. Tierra y mantilo de flora adventicia al borde de un invernadero de tomates, $250 \mathrm{~m}$. 28RES9353433

F-70. Triquivijate, 1-IV-95. Suelo de cultivo abandonado de Opuntia ficus-indica, 223 m. 28RFS007444

F-72. Barranco de Río Cabras, 1-VI-95. Mantillo de Tamarix canariensis con corteza gris, $150 \mathrm{~m}$. 28RES953382

F-74. Morro Velosa, 27-VI-95. Mantillo de Ficus carica, 200 m. 28RES931462

F-75. Morro Velosa, 27-VI-95. Líquenes sobre pared basáltica, 200 m. 28RES 931462

F-76. El Fraile, 21-VII-95. Líquenes (Rochella y Ramalina) sobre paredes de basalto con orientación sur, $560 \mathrm{~m}$. 28RES598072

F-77. El Fraile, 21-VII-95. Suelo y mantillo de Nauplius sericeus muy húmedos en ladera norte, $560 \mathrm{~m}$. 28RES598072

F-78. El Fraile, 21-VII-95. Aglomerado muy húmedo por condensación de niebla en hojas muertas de Nauplius sericeus en ladera norte, 560 m. 28RES598072

F-79. Casas de Cofete, 21-VII-95. Suelo y mantillo de Lycium intricatum en cultivos de secano abandonados, $100 \mathrm{~m}$. 28RES609081

F-80. Madre del Agua, 25-VII-95. Suelo muy húmedo junto a riachuelo con Apium sp. y Juncus acutus, $60 \mathrm{~m}$. 28RES 850419

F-82. Llanos de La Higuera, 25-VII-95. Suelo y mantillo muy secos de Suaeda sp., 30 m. 28RES966255

Los ejemplares han sido separados mediante el método de Berlese-Tullgren y aclarados en ácido láctico caliente.

\section{Resultados}

ESPECIES DETERMINADAS (entre paréntesis número de ejemplares):

Familia COSMOCHTHONIIDAE Grandjean, 1947 Cosmochthonius lanatus (Michael, 1885). F-46 (4); F-61 (1)

Cosmochthonius reticulatus Grandjean, 1942. F-16 (1); F-49 (1)

Familia SPHAEROCHTHONIIDAE Grandjean, 1947 Sphaerochthonius splendidus (Berlese, 1904). F-60 (1); F-65 (1); F-66 (1); F-74 (17); F-77 (1)

Familia PHTHIRACARIDAE Perty, 1841

Calyptophthiracarus canariensis n. sp. F-27 (30); F-31 (1); F-38 (3); F-47 (10); F-67 (1); F-74 (6)
Familia EUPHTHIRACARIDAE Jacot, 1930

Rhysotritia ardua (Koch, 1841). F-37 (5)

Familia LOHMANNIIDAE Berlese, 1916

Lohmannia loebli Mahunka, 1977. F-39 (1); F-68 (8 ad. +2 ninf.)

Reptacarus sagatus Pérez-Iñigo \& Peña, 1996. F-68 (1)

Familia EPILOHMANNIIDAE Oudemans, 1923

Epilohmannia cylindrica cylindrica (Berlese, 1904). F-39 (2)

Familia CAMISIIDAE Oudemans, 1900

Camisia horrida (Hermann, 1804). F-34 (3); F-46 (1 tritoninfa)

Camisia cf. biverrucata (Koch, 1839). F-62 (9)

Familia NOTHRIDAE Berlese, 1896

Nothrus biciliatus Koch, 1841. F-40 (1)

Nothrus cf. palustris Koch, 1839. F-74 (1)

Familia TRHYPOCHTHONIDAE Willmann, 1931

Trhypochthonius tectorum (Berlese, 1816). F-38 (11)

Familia LIODIDAE Grandjean, 1954

Neoliodes ionicus Sellnick, 1932. F-7 (1 ninfa); F-15 (1 ninfa); F-32 (4 ninfas); F-34 (5); F-38 (1)

Familia HERMANNIELLIDAE Grandjean, 1934

Issaniella mograbin Grandjean, 1962. F-17 (3)

Familia ALEURODAMAEIDAE Paschoal \& Johnston, 1984

Aleurodamaeus setosus (Berlese, 1883). F-14 (13); F-25 (29); F-28 (1); F-32 (1); F-37(4); F-65 (3); F-74 (14)

Familia GYMNODAMAEIDAE Grandjean, 1954 Jacotella ornata (Pérez-Iñigo, 1972). F-7 (6); F-8 (1); F-14 (2); F-25 (22); F-26 (6); F-38 (3); F-65 (10); F-77 (13)

Familia PHEROLIODIDAE Paschoal, 1987

Licnoliodes adminensis Grandjean, 1933. F-26 (1); F-43 (6); F-46 (1); F-65 (13); F-70 (1); F-77 (1)

Familia LICNOBELBIDAE Grandjean, 1965

Licnobelba latiflabellata (Paoli, 1908). F-77 (43)

Familia BELBIDAE Willmann, 1931

Subbelba elisae fuerteventurae n. subsp. F-27 (21); F-28 (6) 
Familia CEPHEIDAE Berlese, 1896

Cepheus sp. (ninfas). F-76 (8)

Ommatocepheus crassisetosus n. sp. F-29 (6); F-76 (9)

Familia DAMAEOLIDAE Grandjean, 1965

Fosseremus quadripertitus Grandjean, 1965. F-28 (2); F-61 (1)

Familia ZETORCHESTIDAE Michael, 1898

Belorchestes sectus n. sp. F-16 (2); F-26 (18); F-63 (1); F-77 (13)

Familia LIACARIDAE Sellnick, 1928

Dorycranosus splendens (Coggi, 1898). F-17 (8); F-35 (1); F-80 (1)

Familia XENILLIDAE Woolley \& Higgins, 1966

Xenillus adelae n. sp. F-46 (1); F-54 (10)

Xenillus longipilus $\mathrm{n}$. sp. F-66 (5); F-68 (1)

Xenillus erbanensis n. sp. F-27 (18)

Xenillus pulvillus n. sp. F-7 (6); F-31 (7); F-38 (5); F-47 (1); F-78 (5)

Familia PELOPPIIDAE Balogh, 1943

Ceratoppia bipilis (Hermann, 1804). F-27 (4)

Familia GUSTAVIIDAE Oudemans, 1900

Gustavia fusifer (Koch, 1841). F-27 (8); F-30 (2); F-65 (9); F-74 (6)

Familia CARABODIDAE Koch, 1837

Carabodes purpurarius n. sp. F-7 (37); F-27 (1); F-28 (6); F-29 (15); F-76 (53); F-77 (12)

Odontocepheus elongatus (Michael, 1879). F-38 (1)

Austrocarabodes arrogans Pérez-Iñigo, 1967. F-5 (1); F-7 (5); F-14 (2); F-32 (5); F-34 (7); F-35 (1); F-38 (8); F-52 (2)

Familia TECTOCEPHEIDAE Grandjean, 1954

Tectocepheus velatus (Michael, 1880). F-7 (43); F-9 (1); F-48 (3)

Familia CALEREMAEIDAE Grandjean, 1965

Caleremaeus monilipes (Michael, 1882). F-27 (10); F-28 (1); F-29 (1); F-38 (6); F-61 (1)

Familia OPPIIDAE Grandjean, 1954

Ramusella (Rectoppia) strinatii Mahunka, 1980. F-28 (3); F-65 (6); F-68 (7); F-74 (22)

Multioppia jandiae n. sp. F-28 (4)

Multioppia wilsoni Aoki, 1964. F-39 (2); F-40 (3); F-54 (1); F-60 (8)

Graptoppia (Stenoppia) cf. italica (Bernini, 1973). F-27 (2); F- 28 (5)
Pseudoamerioppia cf. paraguayensis Balogh \& Mahunka, 1981. F-39 (1)

Familia QUADROPPIIDAE Balogh, 1983

Quadroppia cf. pseudocircumita Minguez, Ruiz \& Subias, 1985. F-28 (2)

Familia SUCTOBELBIDAE Jacot, 1938

Suctobelbella acutidens Forsslund, 1941. F-27 (1); F-28 (3)

Familia CYMBAEREMAEIDAE Sellnick, 1928

Scapheremaeus subcorniger n. sp. F-5 (2); F-76 (27)

Scapheremaeus cf. tricarinatus Sitnikova, 1975. F-7 (2); F-28 (2); F-29 (12); F-62 (8)

Familia MICREREMIDAE Grandjean, 1954

Micreremus brevipes (Michael, 1888). F-31 (2)

Familia LICNEREMAEIDAE Grandjean, 1931

Licneremaeus cf. similis Pérez-Iñigo Jr., 1989. F-27 (2); F-28 (1)

Familia PASSALOZETIDAE Grandjean, 1954

Passalozetes africanus Grandjean, 1932. F-52 (1)

Passalozetes inlenticulatus Mihelcic, 1959. F-25 (4); F-26 (2); F-48 (1); F-65 (6)

Passalozetes scholzi n. sp. F-16 (1); F-7 (6); F-63 (6); F-64 (1); F-65 (45); F-70 (1), F-77 (13)

Bipassalozetes reticulatus (Mihelcic, 1957). F-5 (2); F-8 (5); F-69 (53); F-77 (1)

Passalobates asper n. g. et n. sp. F-1 (2); F-2 (1); F-18 (1)

Familia SCUTOVERTICIDAE Grandjean, 1954

Scutovertex sculptus Michael, 1879. F-7 (5); F-27 (2); F-28 (4); F-41 (1); F-61 (18); F-62 (1); F-63 (21); F-72 (1); F-77 (4)

Scutovertex minutus (Koch, 1835). F-5 (15); F-8 (10); F-9 (11); F-10 (16); F-16 (1); F-22 (1); F-32 (10)

Scutoverticosus insperatus $\mathrm{n}$. sp. F-7 (4)

Ethiovertex elisae n. sp. F-5 (2); F-8 (1)

Familia ZETOMOTRICHIDAE Grandjean, 1934

Zetomotrichus lacrimans Grandjean, 1934. F-48 (7); F-49 (1); F-60 (2); F-65 (10); F-68 (71); F-74 (12)

Familia ORIBATULIDAE Thor, 1929

Oribatula tibialis (Nicolet, 1855). F-40 (1)

Oribatula incerta Pérez-Iñigo \& Peña,1996. F-5 (1); F-58 (1); F-66 (1); F-75 (1); F-76 (2)

Zygoribatula frisiae (Oudemans, 1900). F-3 (9); F-5 (5); F-16 (1); F-19 (28); F-22 (62); F-24 (1); 
F-25 (15); F-38 (7); F-43 (12); F-46 (6); F-47 (16); F-49 (6); F-51 (2); F-69 (9); F-74 (13); F-75 (3); F-78 (74)

Zygoribatula exilis (Nicolet, 1855). F-26 (5); F-28 (4); F-77 (9)

Zygoribatula connexa (Berlese, 1904). F-43 (9); F-55 (1); F-59 (1); F-60 (53); F-65 (22); F-66 (8); F-68 (25)

Zygoribatula incomperta n. sp. F-76 (5)

Zygoribatula cf. lenticulata Minguez \& Subias, 1986. F-82 (2)

Dometorina plantivaga (Berl.,1895).F-6 (7);F-44 (2);F-76 (4)

Phauloppia lucorum (Koch, 1840). F-31 (2); F-76 (26)

Familia PROTORIBATIDAE J. Balogh \& P. Balogh, 1984

Fuerteventuria mirabilis n. g. et n. sp. F-26 (6); F-31 (36)

Familia SCHELORIBATIDAE Grandjean, 1953

Hemileius hierrensis Pérez-Iñigo, 1984. F-16 (2); F-25 (12); F-26 (5); F-27 (24); F-30 (1); F-38 (25); F-74 (523); F-75 (10)

Scheloribates fimbriatus Thor, 1930. F-1 (1); F-2 (4); F-39 (3); F-40 (7); F-52 (1); F-60 (10); F-66 (14); F-69 (16)

Scheloribates laevigatus (Koch, 1836). F-40 (3)

Familia ORIPODIDAE Jacot, 1925

Truncopes optatus Grandjean, 1956. F-47 (13); F-66 (1)

Pirnodus soyeri Travé, 1969. F-6 (1 m. y 1 h.); F-15 (6 h.); F-21 (1 h.); F-47 (1 h.); F-55 (1 m. y 7 h.); F- 62 (6 h.); F-67 (1 h.); F-75 (1 h.); F-76 (5 m. y 27 h.)

Familia HAPLOZETIDAE Grandjean, 1936

Lauritzenia (Incabates) depilis n. sp. F-65 (1); F-74 (1)

Familia CERATOZETIDAE Jacot, 1925

Ceratozetes conjunctus Mihelcic, 1956. F-60 (12)

Latilamellobates incisellus (Kramer, 1897). F-5 (2); F-33 (2); F-43 (1); F-54 (2); F-61 (6); F-64 (12); F-65 (12); F-69 (2); F-70 (1)

Familia CHAMOBATIDAE Grandjean, 1954

Chamobates schuetzi (Oudemans, 1902). F-27 (19); F-67 (1)

Familia HUMEROBATIDAE Grandjean, 1970

Humerobates rostrolamellatus Grandjean, 1936. F-7 (5); F-16 (1)
Familia PHENOPELOPIDAE Petrunkevitch, 1955

Eupelops claviger fuerteventurae $\mathrm{n}$. subsp. F-27 (1); F-31 (29); F-76 (1); F-78 (28)

Peloptulus reticulatus Mihelcic, 1957. F-7 (5); F-14 (7); F-16 (1); F-26 (2); F-63 (1); F-77 (2); F-79 (2)

Familia ORIBATELLIDAE Jacot, 1925

Oribatella mahani n. sp. F-27 (1); F-28 (5); F-29 (11)

Familia ACHIPTERIIDAE Thor, 1929

Pseudachipteria agenjoi Pérez-Iñigo, 1976. F-28 (60); F-74 (5)

Familia GALUMNATIDAE Jacot, 1925

Galumna alatum (Hermann, 1804). F-27 (20); F-28 (28); F-30 (9); F-31 (23)

Galumna setigerum Mihelcic, 1956. F-48 (2)

Galumna tarsipennatum Oudemans, 1913. F.60 (8)

Pergalumna cf. myrmophilum (Berlese, 1915). F-37 (8); F-38 (12)

Pilogalumna alliferum longiareatum Pérez-Iñigo, 1976. F-26 (18); F-68 (228)

DESCRIPCIÓN DE LOS NUEVOS TÁXONES.- Todas las dimensiones se expresan en micras métricas (las dimensiones medias entre paréntesis). Se utilizan las siguientes abreviaturas: le: setas lamelares; ro: setas rostrales; in: setas interlamelares; ex: setas exobotrídicas; ss sensilo; N: setas gastronóticas; apo: apodema, G: setas genitales; Ag: setas adgenitales; An: setas anales; Ad: setas adanales. Los holotipos de las especies y subespecies nuevas se conservan montados en Hoyer en la colección del Museo Nacional de Ciencias Naturales (MNCN) en Madrid, expresándose en cada caso la numeración con la que han sido catalogados.

\section{Calyptophthiracarus canariensis n. $\mathrm{sp}$.} (figs. 1-3)

Holotipo: Un ejemplar de F-67 (MNCN 20.02/9001)

Dimensiones: aspis 311-352 (335), notogáster 638-730 $(666,4)$. Toda la cutícula presenta un punteado muy denso, que en los bordes se transforma en fina estriación; no se aprecian foveolas. No existe cresta longitudinal en el áspis, ni estrías en su parte posterior; le e in situadas en la misma línea transversal, las últimas notablemente largas, finas y 


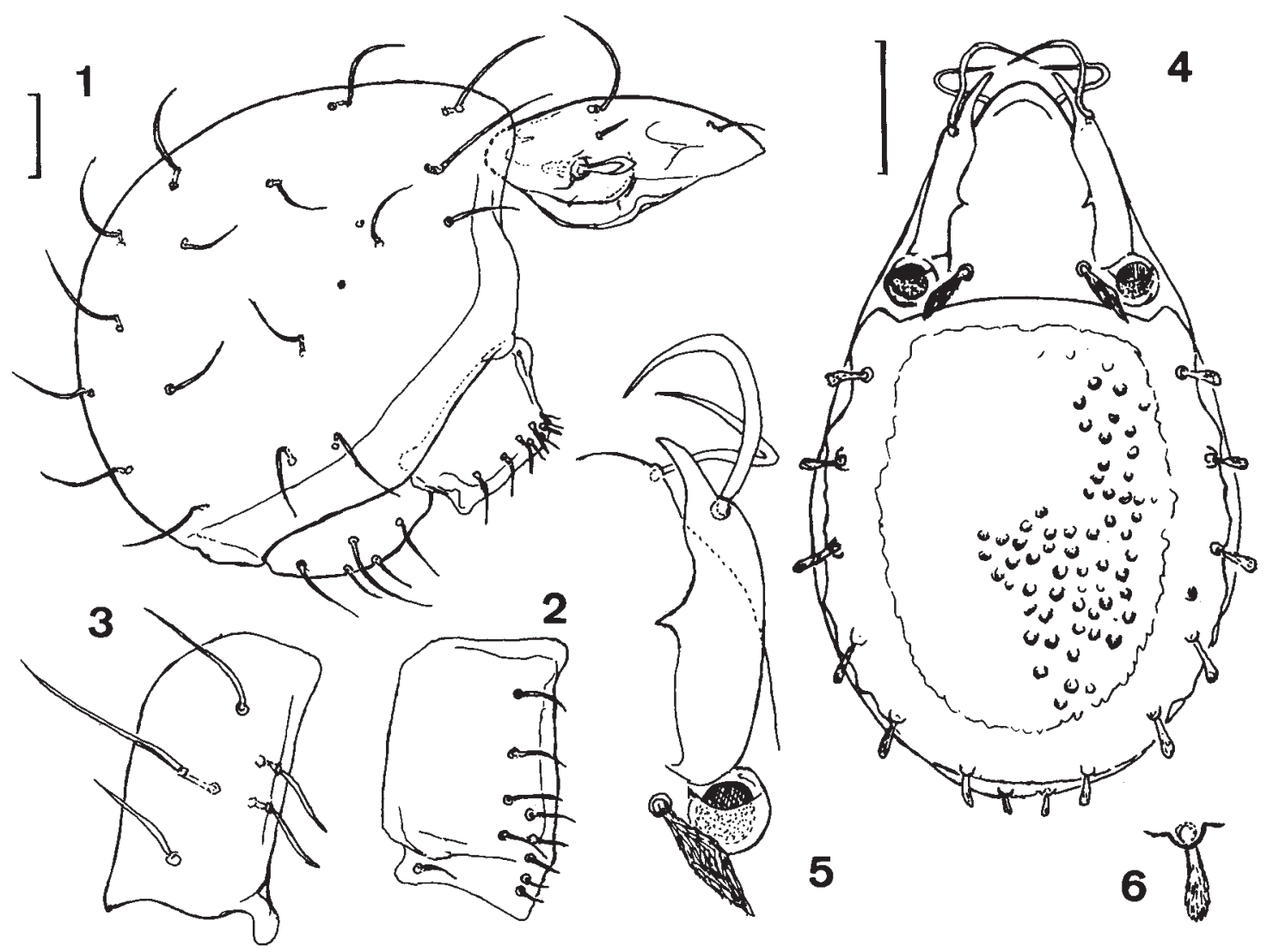

Figs. 1-6.- Calyptophthiracarus canariensis n. sp.: 1) aspecto lateral; 2) valva genito-adgenital; 3) valva ano-adanal. Ommatocepheus crassisetosus n. sp.: 4) aspecto dorsal; 5) lamela y setas rostral, lamelar e interlamelar del lado derecho; 6) seta posterior del notogáster. Escala: $100 \mu \mathrm{m}$.

Figs. 1-6.- Calyptophthiracarus canariensis n. sp.: 1) lateral view; 2) genito-aggenital plate; 3) ano-adanal plate. Ommatocepheus crassisetosus n. sp.: 4) dorsal view; 5) lamella and rostral, lamellar and interlamellar right setae; 6) posterior notogastral seta. Scale $100 \mu \mathrm{m}$.

lisas, de insercion casi perpendicular y curvadas hacia atrás; las le miden $1 / 3$ de las in, rectas, lisas y más finas, terminadas en punta; ro algo más gruesas y largas que las $l e$, casi rectas, insertas lejos del borde. Botridios con escama supero-posterior; ss en forma de maza, con el extremo redondeado formado por una lámina muy fina. Carena lateral larga y poco marcada; ex muy delgada. Notogáster muy abovedado; $\mathrm{N}$ : 17, los tres anteriores $(c 1, c 2$ y $c 3)$ largos, $c 1$ y $c 2$ como in, $c 3$ más corto. Las restantes setas algo más cortas, todas arqueadas y lisas; fisuras ia e im apreciables. G:9, cortas, finas y lisas, dispuestas en dos filas: $g 1-g 5$ en el borde, $g 6-g 9$ más alejadas de éste; $g 6$ queda sobre la seta $g 4$; seta
Ag bien visible. Ad-An: 5, las 3 adanales muy alejadas del borde paraxial, sobre todo la central; todas finas, lisas, largas y algo curvas, la más corta es la anterior; las setas anales, próximas entre sí y en el borde, son notablemente más cortas y finas que las anteriores. Patas monodáctilas, la uña tiene un saliente central en la concavidad; fémur IV con 4 setas, casi en el extremo distal.

Discusión: Se asigna esta especie al género Calyptophthiracarus a causa del punteado de su cutícula y ausencia de foveolas; presencia de N: 17, situación de las setas An-Ad y G, e inserción de la seta in. Tiene cierto parecido con $C$. heterotrichus 
(Mahunka, 1973) de Creta, Crimea, Cáucaso, Turquía y Asia Rusa, pero se diferencia en la talla y forma del sensilo. C. candidulus (Niedbala, 1982), del Cáucaso, presenta setas más cortas, especialmente las in; C. latior (Niedbala, 1982), de India y Extremo Oriente Ruso tiene menor talla y setas le casi vestigiales.

\section{Subbelba elisae fuerteventurae n. subsp.}

$$
\text { Holotipo: Un ejemplar de F-27 (MNCN 20.02/9002) }
$$

Estos ejemplares difieren de la forma típica, de Tenerife, (Pérez-Iñigo, 1976) por: 1) Mayor talla, miden 450-600 x 300-410, mientras que la forma nominada alcanza 351-390 de longitud; los ejemplares de La Gomera (Pérez-Iñigo, 1986) son algo mayores: 395-420 de longitud, también son mayores que los de la subespecie Subbelba elisae grancanariae Pérez-Iñigo \& Peña, 1996 que miden 392-445 x 232-287. 2) Setas N más robustas que en los ejemplares de Tenerife y La Gomera, sin extremo flageliforme, excepto las posteriores, en esto se parece a la subespecie de Gran Canaria. 3) ss más cortos y menos flexuosos que en las otras dos subespecies.

Esta especie parece ser un endemismo canario, con formas próximas pero diferentes en cada una de las islas.

\section{Ommatocepheus crassisetosus n. sp.} (figs. 4-6)

Holotipo: Un ejemplar de F-74 (MNCN 20.02/9003)

Dimensiones: 506-590 $(544,00)$ x 308-374 $(331,71)$. Rostro redondeado con un pequeño saliente a cada lado donde se inserta la seta ro. Lamelas con la parte estrecha de la cúspide relativamente corta, su extremo queda lejos de la del lado opuesto. le muy larga, más del doble que la mencionada parte estrecha, gruesa, curva y puntiaguda, que se cruza con la del lado opuesto; ro anchas, muy largas y terminadas en pico, generalmente dirigidas hacia fuera y posteriormente acodadas hacia dentro hasta casi ponerse en contacto con la del otro lado. Lamela relativamente estrecha en la base, de tal modo que la seta in se inserta fuera de ella; esta seta es gruesa, larga, fusiforme, terminada en punta, de color obscuro y cubierta por numerosas bárbulas cortas. Los ss son los característicos del género. Borde anterior del notogáster ligeramente curvo, con un saliente humeral redondeado a cada lado. N: 9, de los que siete pares se disponen en el dorso en línea próxima al borde, sobre pequeños tubérculos, son setas bastante largas y, sobre todo, gruesas como pequeñas mazas, de cabeza áspera. En el lado ventral se aprecian otros dos pares notablemente menores. Area central del notogáster con tubérculos diseminados. Ningún apodema es completo, salvo el apo 1, el apo 4 es inexistente. Fórmula coxisternal: 2-1-3-2 (?). Aberturas genital y anal separadas por un puente estrecho. G: 6, a lo largo del borde interno de la valva; Ag: 1, Ad: 3 y An: 2. Las setas de estos dos últimos grupos son muy largas y ensiformes. Tarsos tridáctilos casi homodáctilos.

Discusión: O. ocellatus (Michael, 1882) presenta lamelas con la parte estrecha terminal muy larga, tanto que alcanza o se cruza con la del lado opuesto; le proporcionalmente cortas, tanto como la parte estrecha de la lamela; in estrechas y aplanadas; $\mathrm{N}$ finas, no insertas en tubérculos y escultura de la parte central del notogáster con fosetas redondeadas. O. pulcherrimus Berlese, 1913 tiene setas le cortas y curvas, in como pequeños bastoncitos cilíndricos de punta aguda y setas $\mathrm{N}$ como pequeñas varillas no insertas en tubérculos.

Derivatio nominis: Del latin crassus, grueso y setosus, por el grosor de las setas in y gastronóticas.

\section{Belorchestes sectus n. sp.} (figs. 7-9)

Holotipo: Un ejemplar de F-26 (MNCN 20.02/9004)

Dimensiones: $338-374(358,25)$ x 222-258 $(244,75)$. Coloración amarillenta, cutícula lisa. Rostro redondeado, con un saliente a cada lado que corresponde al extremo de la prolamela; ro insertas muy próximas entre sí, casi en contacto, inmediatamente detrás de ellas se extienden las quillas 

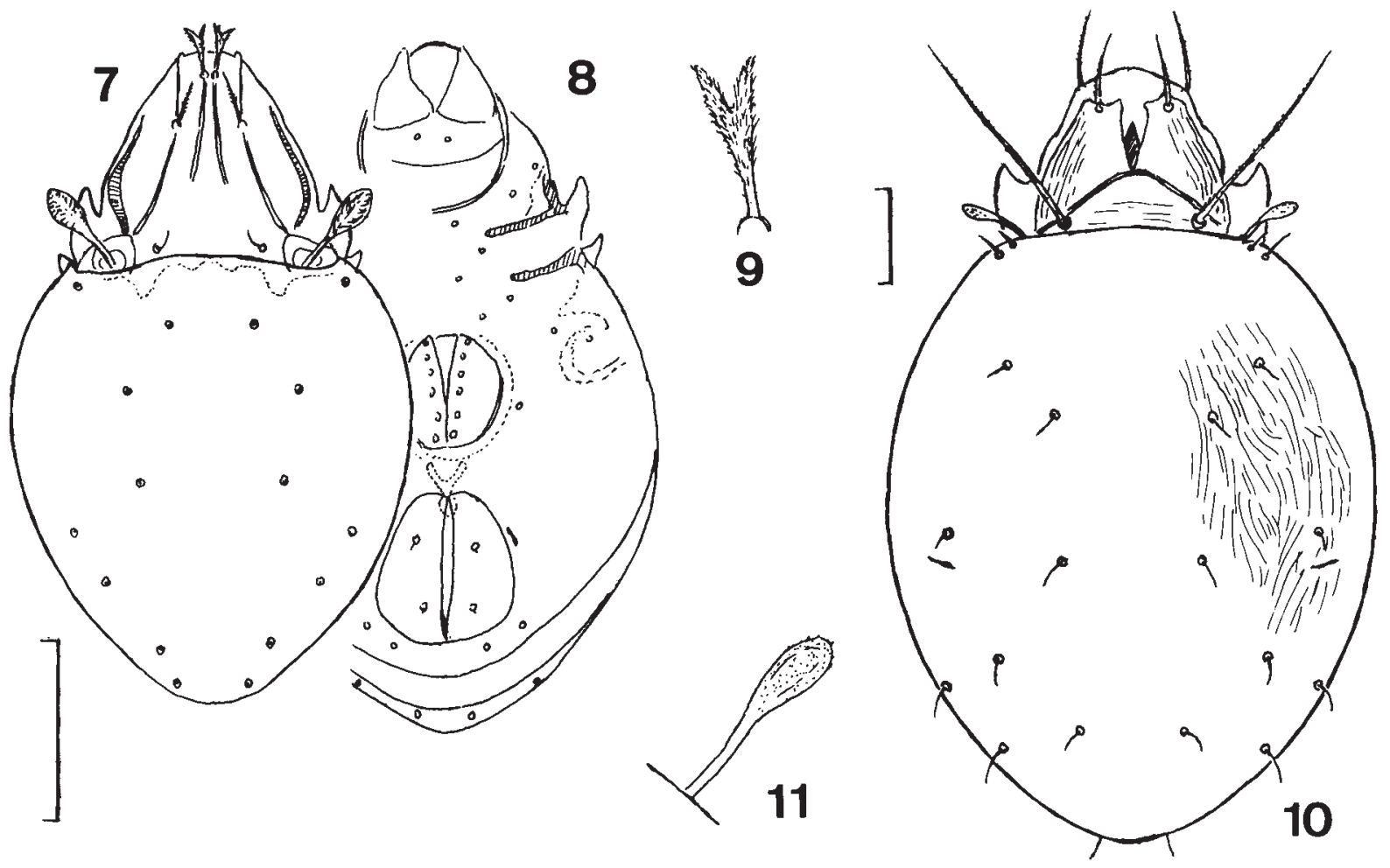

Figs. 7-11.- Belorchestes sectus n. sp.: 7) aspecto dorsal; 8) aspecto ventral, 9) seta rostral. Xenillus adelae n. sp.: 10) aspecto dorsal; 11) sensilo. Escala: $100 \mu \mathrm{m}$.

Figs. 7-11.- Belorchestes sectus $\mathrm{n}$. sp.: 7) dorsal view: 8) ventral view; 9) rostral seta. Xenillus adelae n. sp.: 10) dorsal view; 11) sensillus. Scale: $100 \mu \mathrm{m}$.

mediales, próximas una a otra y bastante largas, divergentes hacia atrás; ro nítidamente bifurcadas y cubiertas de bárbulas cortas. Lamelas lineares, parten de los botridios y terminan en pequeños tubérculos donde se insertan las le, que son finas, rectas y cubiertas con algunas bárbulas y que alcanzan las inserciones de las ro; in muy cortas, finas y lisas, pero fácilmente visibles: ss mazudo y cubierto de bárbulas bastante largas. Foseta rostral muy poco marcada. Notogáster perfectamente separado del prodorso por un surco disyugal nítido y completo. N: 10 (sólo ocho pares visibles dorsalmente), reducidas a los alveolos. Notogáster con frecuencia de forma acorazonada, por estar estrechado en la parte posterior. Proceso humeral bien desarrollado. Sólo se aprecian los apodemas 1, 2 y sj, los últimos muy cortos. Setas coxisternales reducidas a los alveolos, su fórmula parece ser la normal. Orificio genital elíptico. G: 6, reducidas a los alveolos, dispuestas en el borde paraxial, frecuentemente $g 4$ se desplaza hacia el centro de la valva, uni o bilateralmente. Orificio anal mayor que el genital, separado de él por un puente estrecho. An: 2, muy cortas; Ag:1 y Ad: 2. Fisuras iad paralelas al borde anal. Organo preanal fuertemente bilobulado. Lateralmente se observa un tutorio en forma de cresta y un subtutorio semejante pero más corto, unidos ambos en la parte posterior mediante una arista curva. Patas semejantes a las de otras especies del género.

Discusión: B. gebennicus Grandjean, 1957 no presenta ro bifurcadas, las carenas mediales sobrepasan por los lados las inserciones de las ro y, sobre todo, no presenta surco disyugal, además tiene 
setas $\mathrm{N}$ finas pero visibles y el tutorio es laminar. $B$. planatus Grandjean, 1951 presenta las quillas mediales del prodorso muy separadas entre sí, lamelas más gruesas, setas rostrales simples, barbuladas y también carece de surco disyugal.

Derivatio nominis: Del latin sectus, cortado, por la presencia de un surco disyugal completo.

Xenillus adelae n. sp. (figs. 10-11)

Holotipo: Un ejemplar de F-54 (MNCN 20.02/9005)

Dimensiones: $814-880(850,66)$ x 484-594 $(524,33)$. Rostro redondeado con un pequeño saliente a cada lado; lamelas anchas, con dos dientes cuspidales poco desarrollados; por lo general, el interno más desarrollado pero existe notable variabilidad, el externo rara vez es puntiagudo; mucrón interlamelar bien desarrollado. Setas ro rectas y provistas de bárbulas cortas; le insertas entre los picos cuspidales, largas $(125 \mu \mathrm{m})$, bastante gruesas, rectas, y cubiertas de bárbulas muy cortas; in insertas casi en el surco disyugal, rectas, dirigidas hacia adelante y afuera, muy largas (220 $\mu \mathrm{m})$ y cubiertas de bárbulas; Superficie lamelar con marcada estriación longitudinal en su parte externa. Botridios cubiertos por el borde anterior del notogáster; $s s$ con tallo fino y cabeza ovalada cubierta de espinitas; miden $55 \mu \mathrm{m}$. Area interlamelar con algunas finas estrías tranversales. Borde anterior del notogáster casi recto; $\mathrm{N}$ : 11, muy finas, lisas, cortas y difíciles de ver; seta humeral anterior casi virtual, la posterior más visible. Superficie de aspecto liso, en realidad cubierta de un punteado muy fino y de estrías muy estrechas de disposición irregular, pero preferentemente longitudinal. Tutorio muy desarrollado que llega a la inserción de ro, sin punta libre; pedotecto I con un ángulo en el borde superior. Apodemas 1, 2, sj y 4 completos, apo 3 reducido; setas coxisternales bastante largas, de fórmula banal; superficie epimérica con un foveolado muy marcado. Orificio genital redondeado y relativamanete pequeño; G: 5 , semejantes a las coxisternales. Orificio anal muy alejado del genital; Ag: 1, An: 2; Ad: 3, todas bastante largas finas y rectas. Quillas ventales de los fému- res poco desarrolladas; tarsos tridáctilos casi homodáctilos.

Discusión: Las setas gastronóticas, gran longitud de las setas in, forma de tutorios y pedotectos y caracteres ventrales aproximan esta especie a $X$. moyae Pérez-Iñigo \& Peña, 1994, de Gran Canaria, pero ésta especie es de menor talla (594-814), con cúspides lamelares de forma diferente, ss más largos con cabeza fusiforme y notogáster y superficie ventral sin estrías sino con poros pequeños y diseminados.

Derivatio nominis: Dedicamos esta especie a Adela Rivero, esposa del segundo de los autores.

Xenillus longipilus $\mathrm{n}$. sp. (figs. 12-14)

Holotipo: Un ejemplar de F-66 (MNCN 20.02/9008)

Dimensiones: 946-1000 (974,40) x 472-616 $(553,33)$. Coloración castaño-amarillenta. Capa cerotegumentaria difícil de desprender. Rostro de apariencia redondeada, pero en realidad ondulado, ro insertas en pequeños nódulos próximos al borde, en el extremo del tutorio, bastante largas (85 micras), dirigidas hacia delante y con espinas cortísimas. Lamelas relativamente cortas y estrechas, la parte más ancha corresponde a las cúspides, que presentan un diente interno redondeado y una superficie curva externa, pues no existe pico externo; cúspides de ambas lamelas en contacto, sólo separadas en su parte posterior por un mucrón pequeño. Setas le no insertas en el extremo sino en la superficie superior de las cúspides, largas (110 $\mu \mathrm{m})$, algo curvas hacia abajo y casi lisas; in insertas casi en el surco disyugal, muy largas $(200 \mu \mathrm{m})$, con cortísimas espinitas en su mitad distal. Botridios casi totalmente cubiertos por el borde gastronótico; ss con tallo que se ensancha paulatinamente en maza de extremo redondeado, que lleva un corto número de espinitas muy pequeñas Superficie interlamelar lisa. Pedotecto I muy desarrollado. Tutorio largo y estrecho. Notogáster ovalado, cutícula con fosetas redondeadas poco marcadas y separadas entre sí. N: 11, muy largas, la más corta es la seta humeral anterior que mide unas 80 


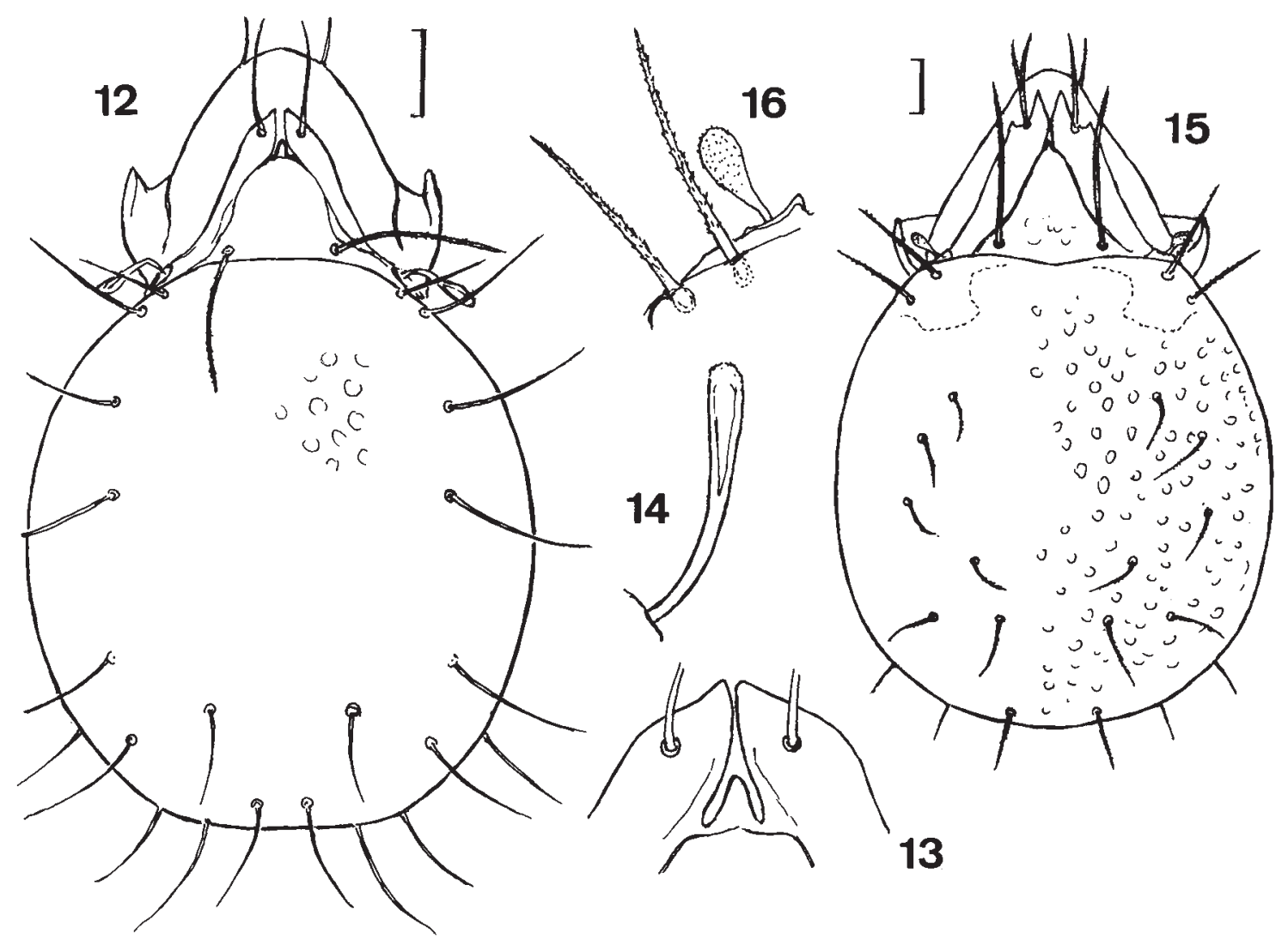

Figs. 12-16.- Xenillus longipilus n. sp.: 12) aspecto dorsal; 13) cúspides lamelares; 14) sensilo. Xenillus erbanensis n. sp.: 15) aspecto dorsal; 16) sensilo y setas humerales. Escala: $100 \mu \mathrm{m}$.

Figs. 12-16.- Xenillus longipilus n. sp.: 12) dorsal view: 13) lamellar cusps;14) sensillus. Xenillus erbanensis n. sp.: 15) dorsal view: 16) sensillus and shoulder setae. Scale: $100 \mu \mathrm{m}$.

$\mu \mathrm{m}$, la posterior alcanza, 150, las restantes entre 150 y 180 . Setas coxisternales de longitud mediana, ligeramente barbuladas, de fórmula banal; apodemas 1,2 , sj completos; los 3 y 4 muy poco desarrollados; superficie coxisternal con fosetas semejantes a las dorsales. G: 5, largas y finas. Orificio genital redondeado y pequeño. Valvas genitales obscuras. Orificio anal considerablemente mayor que el genital; An: 2, Ag: 1, Ad: 3, estas últimas largas. Superficie anogenital lisa; valvas anales con unas pocas fosetas poco señaladas. Patas tridáctilas homodáctilas. Fémures de todas las patas con quillas poco desarrolladas.

Discusión: La forma de las lamelas y la inserción de las setas le es muy semejante a la de $X$. disjunctus Balogh \& Mahunka, 1977, de Chile, pero en esta especie no existe mucrón y las setas gastronóticas son más gruesas, rígidas y barbuladas. Se conocen algunas especies con setas gastronóticas largas, pero ninguna de ellas presenta simultáneamente la misma forma de las cúspides lamelares, implantación de le y forma del sensilo.

Derivatio nominis: Del latín longus: largo y pilus: pelo, cabello.

Xenillus erbanensis n. sp. (figs. 15-16)

Holotipo: Un ejemplar de F-27 (MNCN 20.02/9006) 
Dimensiones: $968-1.380(1.150,33)$ x 638-880 $(805,50)$. Color castaño, capa cerotegumentaria gruesa y difícil de quitar. Rostro redondeado, de borde irregular. Setas ro ligeramente curvas con bárbulas muy cortas. Cúspides lamelares con dos dientes, el interno más desarrollado, entre ambos se inserta le que es gruesa, recta, dirigida hacia delante, cubierta de bárbulas cortas y de unas 120 $\mu \mathrm{m}$ de longitud. Espacio intercuspidal estrecho, más ancho en la parte posterior, donde existe un mucrón pequeño. Setas in gruesas, más largas que las le pero del mismo aspecto. Botridios cubiertos parcialmente por el borde anterior del notogáster; ss con tallo fino y corto y cabeza ovalada con algunas espinitas. Superficie interlamelar con punteado muy fino y algunas fosetas en la parte posterior. Pedotecto I grande, sin ángulo superior. Tutorio largo. Borde anterior del notogáster ligeramente cóncavo; setas humerales largas, las más largas del notogáster $(155 \mu \mathrm{m})$, gruesas, rectas y barbuladas, sobrepasan con mucho el extremo del sensilo; las restantes, en número y disposición normales, tienen el mismo aspecto que las humerales pero son algo más cortas. Superficie gastronótica con punteado fino y foveolas poco marcadas. Apodemas 1, 2 y sj completos, 3 reducido y 4 casi inexistente. Setas coxisternales bastante largas, de fórmula normal. G: 5, finas y lisas; Ag: 1; An: 2 y Ad: 3, ligeramente barbuladas. Tarsos tridáctilos, casi homodáctilos. Fémures con láminas ventrales poco desarrolladas.

Discusión: Notogáster con setas humerales más largas que las restantes y sensilo corto y mazudo son caracteres que diferencian esta especie de otras del mismo género.

Derivatio nominis: De Erbania, nombre con el que se conocía a Fuerteventura en las crónicas normandas.

Xenillus pulvillus n. sp. (figs. 17-18)

Holotipo: Un ejemplar de F-7 (MNCN 20.02/9007)

Dimensiones: 880-1.100 $(1.027,71)$ x 594-836 $(718,57)$. Cerotegumento grueso y difícil de qui- tar. Rostro amplio, redondeado, de borde irregular; ro muy separadas entre sí, arqueadas y rugosas; lamelas terminadas en cúspides con un diente interno bien desarrollado y sin diente externo; le gruesa, recta, áspera, de unas $160 \mu \mathrm{m}$ de longitud, inserta en el borde anterior, por fuera del diente. Espacio intercuspidal ancho, de lados paralelos, con un pequeño mucrón en el fondo; in muy largas $(270 \mu \mathrm{m})$, ásperas, rectas y dirigidas hacia delante y afuera. Espacio interlamelar con punteado denso y algunas foveolas. Botridios casi totalmente ocultos por el borde gastronótico; ss muy cortos, ovoideos, el cortísimo tallo y la parte proximal de la cabeza no sobresalen del botridio. Superficie gastronótica con foveolas redondeadas, dispersas y poco marcadas. $\mathrm{N}$ : 11 , los dos pares humerales gruesos, ásperos y rectos, aunque cortos sobresalen mucho más que el botridio; los restantes pares algo más largos, más finos, también ásperos. Tutorio largo y estrecho. Sólo apo $1 \mathrm{com}-$ pleto, apo 2 y apo $\mathrm{sj}$, bastante desarrollados, no llegan al centro, apo 3 muy corto y apo 4 ausente. Setas coxisternales bastante desarrolladas, de fórmula normal. Superficie epimeral cubierta de fosetas redondeadas. G: 5, Ag: 1, An: 2 y Ad: 3, como las coxisternales o más largas; iad algo separada del borde anal, pero paralela al mismo. Tarsos tridáctilos casi homodáctilos. Fémur I sin quilla, los restantes tienen quilla ventral sólo en el extremo distal del artejo.

Discusión: X. clypeator Robineau-Desvoidy, 1839 presenta ss parecidos a los de la nueva especie, pero las lamelas son diferentes y las setas in muy cortas casi vestigiales. Ninguna otra especie presenta reunidos los caracteres de ss, lamelas y setas in que muestra la nueva especie.

Derivatio nominis: Del latín pulvillus: almohadilla.

Carabodes purpurarius n. sp. (figs. 19-22)

Holotipo: Un ejemplar de F-77 (MNCN 2002/9009)

Dimensiones: Hembras 484-560 $(518,40) \mathrm{x}$ 308-400 (342,60); machos 400-453 (423,20) x 


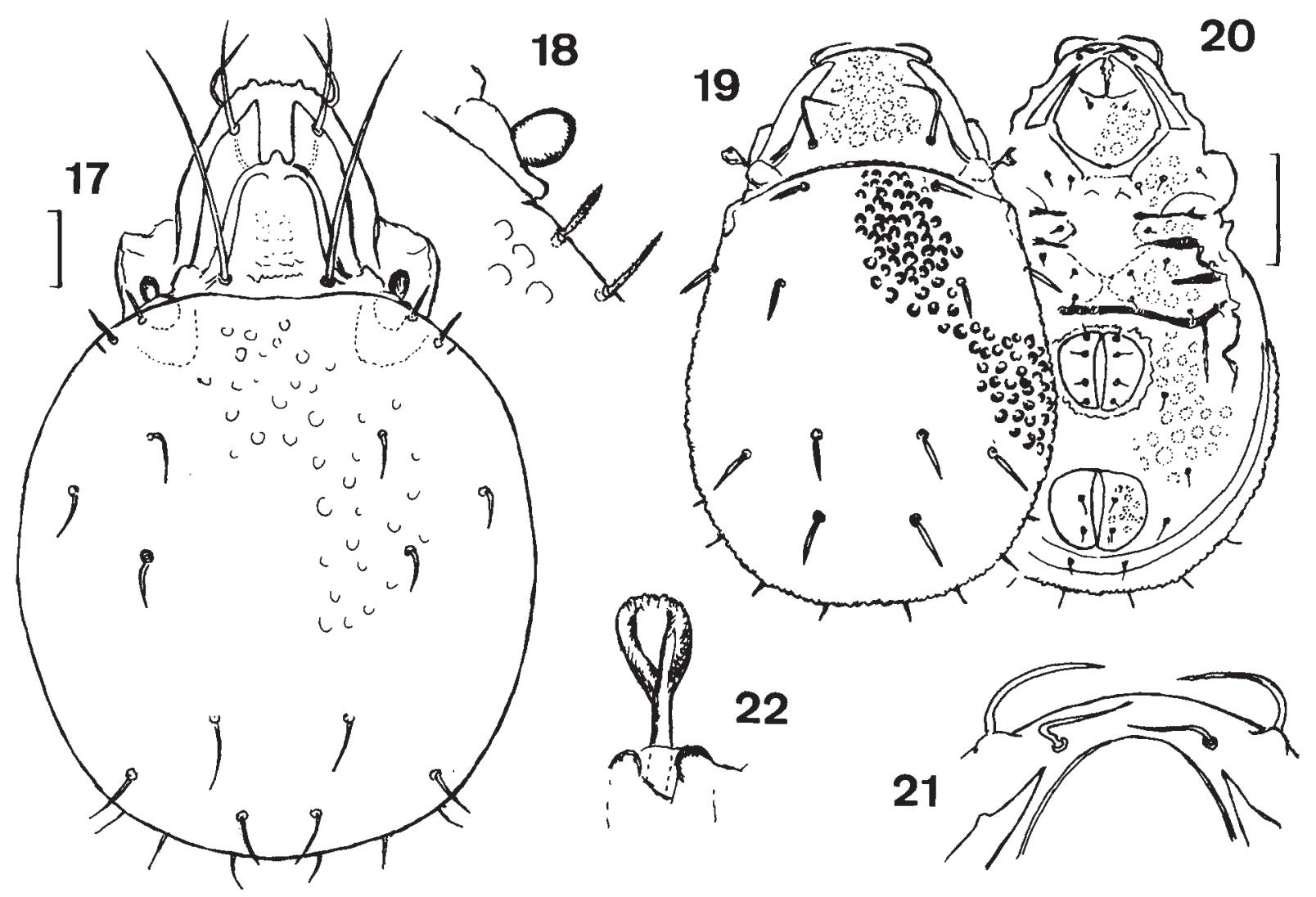

Figs. 17-22.-Xenillus pulvillus n. sp.: 17) aspecto dorsal; 18) sensilo y setas humerales. Carabodes purpurarius $\mathrm{n}$. sp.: 19) aspecto dorsal; 20) aspecto ventral; 21) visión ventral del rostro; 22) sensilo. Escala $100 \mu \mathrm{m}$.

Figs. 17-22.- Xenillus pulvillus $\mathrm{n} . \mathrm{sp} .:$ 17) dorsal view: 18) sensillus and shoulder setae. Carabodes purpurarius $\mathrm{n} . \mathrm{sp} .:$ 19) dorsal view; 20) ventral view; 21) vental view of the rostrum; 22) sensillus. Scale $100 \mu \mathrm{m}$.

250-338 (287,00). Coloración castaña, cerotegumento fino. Escultura prodorsal formada por fosetas irregulares, separadas por líneas más elevadas, que forman un retículo poco saliente. Hipostoma, región coxisternal, placa ventral y valvas anales cubiertas por fosetas redondeadas. Notogáster con tubérculos redondeados , obscuros, sin tendencia a confluir; valvas genitales lisas. Rostro amplio y redondeado; lamelas de inserción muy lateral, poco elevadas, más obscuras que el resto. No hay translamela; ro insertas en el lado ventral del rostro, delante del extremo del tutorio, finas, lisas y fuertemente acodadas hacia dentro, no visibles dorsalmente; le curvas, dirigidas hacia dentro, más gruesas y largas que las anteriores, insertas casi en el ápice de las lamelas; in lisas, algo gruesas y curvas, insertas por dentro de las lamelas. Botridio con una profunda escotadura ventral; ss del tipo de las especies del grupo "minusculus"; tutorio laminar estrecho y largo. Borde anterior del notogáster arqueado; N: 10, lisas y rectas, los seis pares dorsales bastante largos (40-45 $\mu \mathrm{m})$, las setas marginales más cortas, todas ligeramente ensanchadas en la parte central y terminadas en punta; salientes humerales poco pronunciados. Apo 1 completo, apo 2 y sj incompletos, apo 3 muy corto, apo 4 completo, describe una amplia curva delante del orificio genital. Setas coxisternales muy finas, lisas y cortas, de fórmula 3-1-3-3. Orificio genital con un marco quitinoso; G: 4; An: 2; Ag: 1 y Ad: 3, todas finas y lisas. Tarsos monodáctilos. 
Discusión: Esta especie pertenece al grupo "minusculus", en el que encontramos siete u ocho especies de distribución sudeuropea y norteafricana; se diferencia de las restantes especies por 1) talla, es mayor que C. dissimilis Bernini, 1976, hasta ahora la mayor del grupo; 2) setas gastronóticas: semejantes a las de $C$. pulcher Bernini, 1976 y $C$. dissimilis pero en estas especies las setas in son más cortas que en la nueva especie. Además de las localidades indicadas en Fuerteventura, esta especie ha sido encontrada en una muestra de suelo procedente de Haría, en Lanzarote, donada por el Dr. E. Ortiz, y que figuraba en la colección del Dr. Pérez-Iñigo sin determinar.

Derivatio nominis: De las islas purpurarias de los fenicios (Lanzarote y Fuerteventura), donde acudían a recoger la púrpura.

Multioppia jandiae n. sp. (figs. 23-25)

Holotipo: Un ejemplar de F-28 (MNCN 20.02/9010)

Dimensiones: 276-294 (287,25) x 125-151 $(137,75)$. Rostro redondeado y entero; ro fuertemente acodadas y barbuladas en su parte distal; le finas y lisas, situadas en el centro del prodorso, una fina línea curva atraviesa el prodorso delante de sus inserciones. Botridios circulares; ss con un tallo bastante largo y una cabeza mazuda y algo apuntada, desprovista de bárbulas, sólo con un corto número de espinitas en el borde; in ligeramente barbuladas, algo más largas que las le. Existen dos pares de manchas claras interlamelares. Notogáster ovalado; N: 12, largas y finas; fisura im situada delante de la seta $l m$. Superficie epimeral con grandes alveolos; Apo 2 corta y apo 3 ausente, las restantes completas. Setas coxisternales cortas y finas, de fórmula 3-1-3-3. Orificios genital y anal muy separados; G: 5, muy cortas; Ag: 1, An: 2 y Ad: 3; ad 1 postanal; $a d 2$ paraanal y $a d 3$ preanal; iad paraanal, ligeramente delante de $a d 2$.

Discusión: Por la situación de im esta especie pertenece al grupo de M. excisa Moritz, 1971. Ninguna otra especie de este género presenta el sensilo desprovisto de bárbulas; $M$. pulchra
Littlewood \& Wallwork tiene un sensilo con escasas bárbulas, pero sus setas gastronóticas son mucho más cortas.

Derivatio nominis: De Jandia, península al sur de Fuerteventura con notables valores naturales.

Scapheremaeus subcorniger $\mathrm{n}$. sp. (figs. 26-29)

Holotipo: Un ejemplar de F-76 (MNCN 20.02/9011)

Dimensiones: 427-481 $(464,80)$ x 249-267 $(263,00)$. Rostro redondeado y entero, prodorso subtriangular, setas ro lisas y algo curvas, setas $l e$ más cortas y situadas próximas entre sí en el centro del prodorso. Sensilos con tallo corto y cabeza subesférica, granulosa. Setas in lisas y de mayor longitud que las ro, de extremo fino, incluso flexuoso. Pedotectos bien desarrollados. Superficie prodorsal cubierta de arrugas quitinosas irregulares. Notogáster ovalado, con los ángulos humerales muy marcados y provistos cada una de una prolongación corniforme. Superficie notogástrica dividida en dos regiones, central y marginal. La central con una escultura de alveolos grandes y poco marcados; en la parte anterior de la marginal se encuentra una lentícula, de bordes poco precisos y en la posterior una pequeña área de forma más o menos circular, bien delimitada, cuya superficie presenta arrugas irregulares pero no alveolos. El resto de la zona marginal con líneas obscuras de disposición radial. Las setas gastronóticas, finas y puntiagudas, bastante largas, son 14 pares. En el lado ventral se aprecian las setas coxisternales, pequeñas pero bien visibles, de fórmula 3-1-3-3. G: 6, An:2, Ag: 1 y Ad: 3, finas y lisas. La escultura de la región ventral está formada por bandas quitinosas entrecruzadas de forma irregular; las valvas anales presentan algunas arrugas longitudinales.

Discusión: Especie que, a primera vista, se parece mucho a S. corniger (Berlese, 1908) de la que se diferencia por: 1) Mayor talla; 2) Setas prodorsales y gastronóticas finas, lisas y mucho más largas que en la especie de Berlese; 3) escultura de la región central, con alveolos poco pronunciados en la especie canaria, prácticamente ausentes en la pequeña 


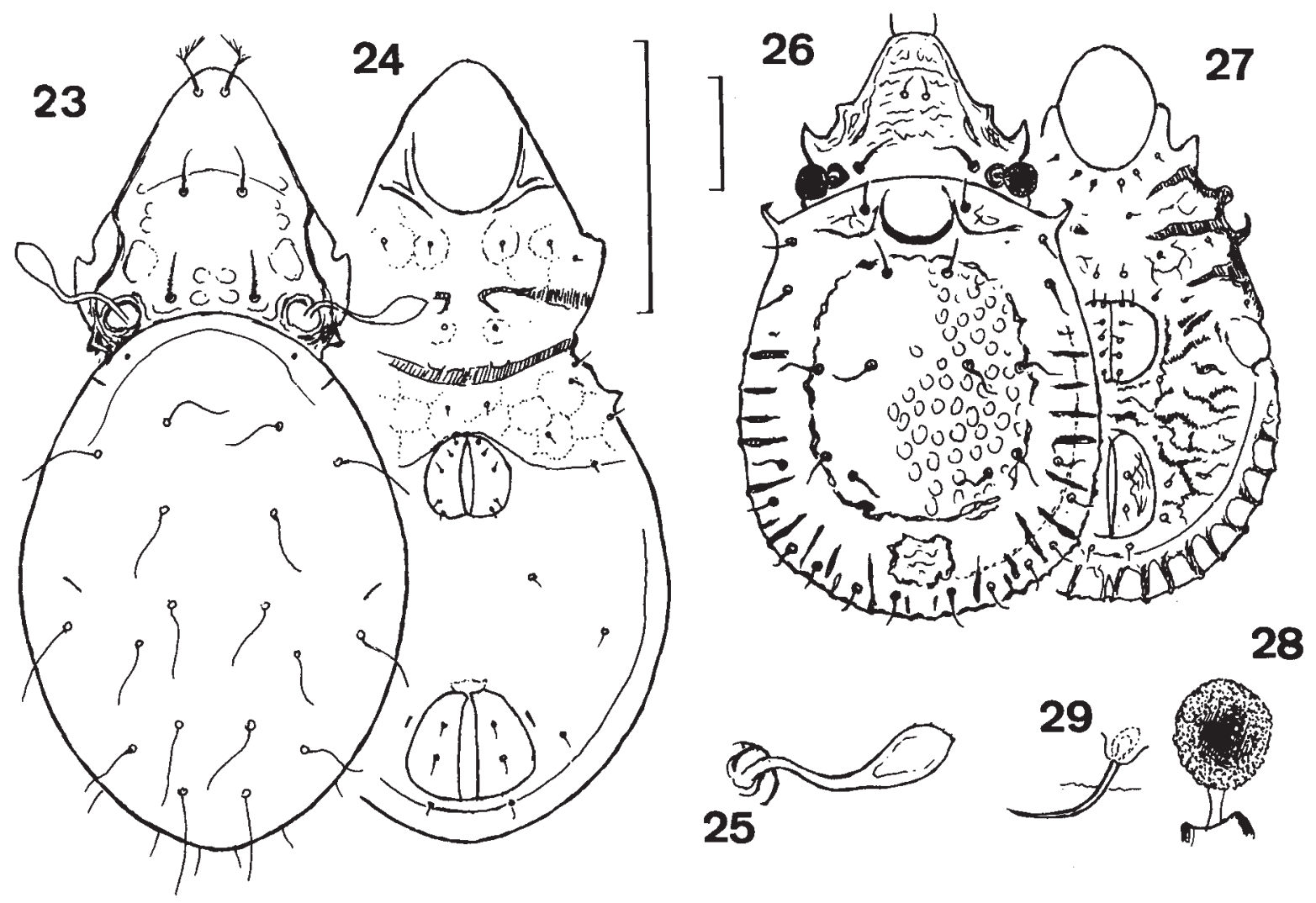

Figs. 23-29.- Multioppia jandiae $\mathrm{n} . \mathrm{sp} .:$ 23) aspecto dorsal; 24) aspecto ventral; 25) sensilo. Scapheremaeus subcorniger $\mathrm{n}$. sp.: 26) aspecto dorsal; 27) aspecto ventral; 28) sensilo; 29) seta del borde posterior. Escala: $100 \mu \mathrm{m}$.

Figs. 23-29.- Multioppia jandiae n. sp.: 23) dorsal view; 24) ventral view; 25) sensillus. Scapheremaeus subcorniger n. sp.: 26) dorsal view; 27) ventral view; 28) sensillus; 29) seta of the posterior edge. Scale: $100 \mu \mathrm{m}$.

área posterior; 4) región ventral con un retículo de mallas anchas e irregulares, diferente de las bandas irregulares de $S$. corniger, valvas anales sin fosetas; y 5) ss menos obscuro, más granuloso que en la especie clásica.

Derivatio nominis: Indica su parecido con $S$. corniger.

\section{Passalozetes scholzi in. sp. (figs. 30-34)}

Holotipo: Un ejemplar de F-16 (MNCN 20.02/9012)

Dimensiones: $356-392(375,62)$ x 178-205 $(188,00)$. Rostro ampliamente redondeado; ro y le finas e incurvadas hacia dentro. Una línea arqueada cruza el prodorso, separando dos áreas, una anterior con escultura poco señalada de líneas irregulares, y otra posterior con escultura semejante a la gastronótica. La parte posterior del prodorso está cruzada por una cresta irregular, detrás de la cual no existe escultura; in cortas y lisas pero fácilmente visibles; $s$ formados por una seta gruesa y áspera, desprovista de bárbulas, que se va afinando hacia el extremo. Notogáster ovalado; el borde anterior avanza sobre el prodorso como es normal en el género y su parte central es borrosa. Lentícula redondeada, grande y bien delimitada; se aprecian bien tres pares de áreas porosas redondas y pequeñas; N: 10, muy cortas, finas y difíciles de ver; fisuras $\mathrm{im}$ bien desarrolladas. Escultura de prodorsum, notogáster, superficie ventral, valvas genitales, anales e hipostoma, formada por tubérculos planos y anchos, encajados unos en otros como las piezas de un puzzle; la separación 


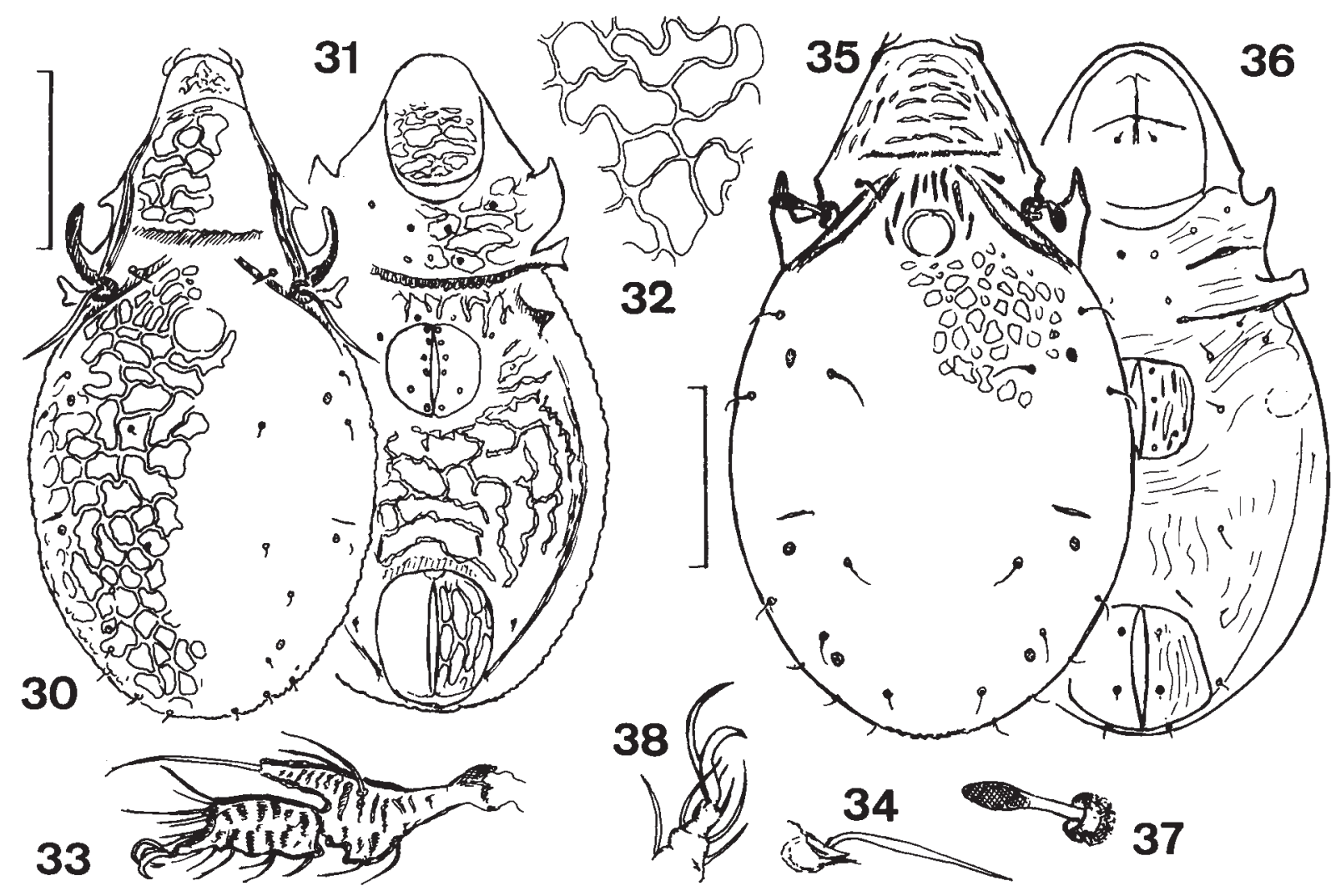

Figs. 30-38.- Passalozetes scholzi n. sp.: 30) aspecto dorsal; 31) aspecto ventral; 32) escultura gastronótica; 33) tibia y tarso de la pata I, vista lateral; 34) sensilo. Passalobates asper n. g. et n. sp.: 35) aspecto dorsal; 36) aspecto ventral) 37) sensilo; 38) uñas de la pata IV derecha. Escala: $100 \mu \mathrm{m}$.

Figs. 30-38. Passalozetes scholzi n. sp.: 30) dorsal view; 31) ventral view; 32) notogastral sculpturing; 33) tibia and tarsus of leg I, lateral view: 34) sensillus. Passalobates asper n. g. et n. sp.: 35) dorsal view; 36) ventral view; 37) sensillus; 38) claws of the right leg IV. Scale: $100 \mu \mathrm{m}$.

entre estos tubérculos es muy estrecha por lo que simula un retículo irregular. Pedotectos I y II muy grandes, cubren totalmente los correspondientes acetábulos. Sólo apo 1 y apo sj desarrollados; setas coxisternales cortas y finas, de fórmula difícil de determinar, pero parece ser 2-1-2-2. Orificios genital y anal muy separados entre sí; G: 6 , reducidas al los alveolos; Ag: 1, An: 2 y Ad: 2; iad delante y bastante alejadas del orificio anal. Tarsos tridáctilos muy heterodáctilos. Tibia I con un solenidio, bastante largo en el extremo de una apófisis muy desarrollada.

Discusión: Hasta donde llegan nuestros conocimientos no existe ninguna otra especie con una escultura como la descrita.
Derivatio nominis: Esta especie ha sido dedicada a D. Stephan Scholz, por su inestimable ayuda en la recolección de muestras y en la determinación de líquenes.

\section{Género Passalobates n. g.}

Familia Passalozetidae. Se caracteriza por presentar setas ro y le insertas al mismo nivel; sensilos cortos y mazudos; valvas genitales con cuatro pares de setas y tarsos bidáctilos, con la uña externa mucho más largas y delgada que la interna. 
Especie tipo: Passalobates asper n. sp. A este género pertenece también Passalozetes sabulosus Shtanchaeva, 1986.

\section{Passalobates asper n. sp. (figs. 35-38)}

\section{Holotipo: Un ejemplar de F-1 (MNCN 20.02/9013)}

Dimensiones: 356-392 (371,75) x 214-231 $(220,25)$. Rostro ancho y redondeado; ro y le finas, lisas, curvas e insertas al mismo nivel; in bastante desarrolladas, lisas y finas. Prodorso cruzado por una cresta más o menos marcada; delante de dicha cresta existe una escultura poco marcada de líneas transversales en el centro y longitudinales a los lados; ss con tallo corto y cabeza mazuda, fusiforme y obscura. Pedotectos muy desarrollados. Notogáster ovalado, más bien ancho (índice longitud/anchura: 1,29). Lentícula redonda bien delimitada. Escultura gastronótica poco marcada, formada por tubérculos planos o manchas alargadas e irregulares, sin tendencia a confluir, que dejan espacios anchos entre ellas. Areas porosas pequeñas pero bastante grandes para lo común en la familia. N: 10, finas y arqueadas. Hipostoma y región esternal con escultura de bandas tranversales irregulares. Setas coxisternales muy finas, de fórmula 3-1-3-2. Orificio genital grande, valvas con bandas anchas longitudinales. G: 4, de ellas $g 3$ fuera del alineamiento de las otras. Valvas anales con bandas longitudinales; An: 2; Ad: 2; Ag: 1; iad delante del orificio anal, algo oblicuas. Superficie ventral con bandas poco marcadas, transversales detrás del orificio genital y más o menos longitudinales en el resto. Tarsos bidáctilos, la uña interna es normal, la externa es mucho más fina y más larga que la interna.

Discusión: Shtanchaeva (1986) describió en Daguestán (norte del Cáucaso) un pasalozétido que denominó Passalozetes sabulosus, que presenta cierta semejanza con la nueva especie, pero que tiene ss de cabeza más alargada y tallo más corto, notogáster con setas falciformes y escultura que, aunque parecida, está más marcada con tendencia a formar en los bordes bandas paralelas.
Derivatio nominis: Del latin asper: áspero, escabroso.

\section{Scutoverticosus insperatus n. sp. (figs. 39-42)}

\section{Holotipo: Un ejemplar de F-7 (MNCN 20.02/9014)}

Dimensiones: 400-420 x 220-230. Capa cerotegumentaria gruesa y difícil de quitar, que lleva adheridas partículas diversas. Rostro redondeado, a cada lado existe un pequeño tubérculo, que se aprecia mejor en observación ventral, donde se inserta ro, que es muy fina, corta y acodada hacia dentro. Lamelas muy anchas, de superficie estriada, con un pico romo en su extremo anterior donde se inserta $l e$, lisa, angulada hacia dentro y tan larga como ro; las lamelas se estrechan hacia atrás, hasta llegar al botridio; no existe translamela, entre ellas queda un espacio alargado y estrecho. Botridios laterales con una pequeña lámina unida al extremo de la lamela. No se aprecian setas in; ss con tallo relativamente corto y cabeza mazuda cubierta por abunantes espinas cortas. Superficie prodorsal lisa. Notogáster sin borde anterior nítido, sustituido por líneas irregulares poco marcadas entre las que se aprecia un área cuadrangular que puede representar una lentícula. Superficie gastronótica lisa; N: 10, muy cortas y lisas, $t a$ y te insertas en pequeños tubérculos, $r l$ y $r 2$ en tubérculos bastante notables y ps 1 y ps 2 en grandes tubérculos en el borde posterior; im bien visible, ia de dirección paralela al borde. Tutorio bastante bien desarrollado, con una base larga, punta grande y parte laminar estriada longitudinalmente. Pedotectos I y II muy desarrollados, el último de extremo bifurcado. Apodemas incompletos, salvo apo 1; apo sj es el mas largo; superficie epimeral con escultura de fosetas irregulares y setas cortas y finas, cuya fórmula parece ser 3-1-3-1. G: 6 , en fila, los dos primeros bien desarrollados y los restantes muy reducidos. Ag: 1; An: 2 y Ad: 3, cortas y finas; $a d l$ y $a d 2$ postanales sobre pequeños tubérculos; ad 3 paraanal; iad palela al borde anal, muy adelantada, casi preanal. Tarsos monodáctilos; fémures con quillas ventrales muy desarrolladas, sobre todo los posteriores; todas las tibias presentan un tubérculo corto y cónico donde se inserta el solenidio. 


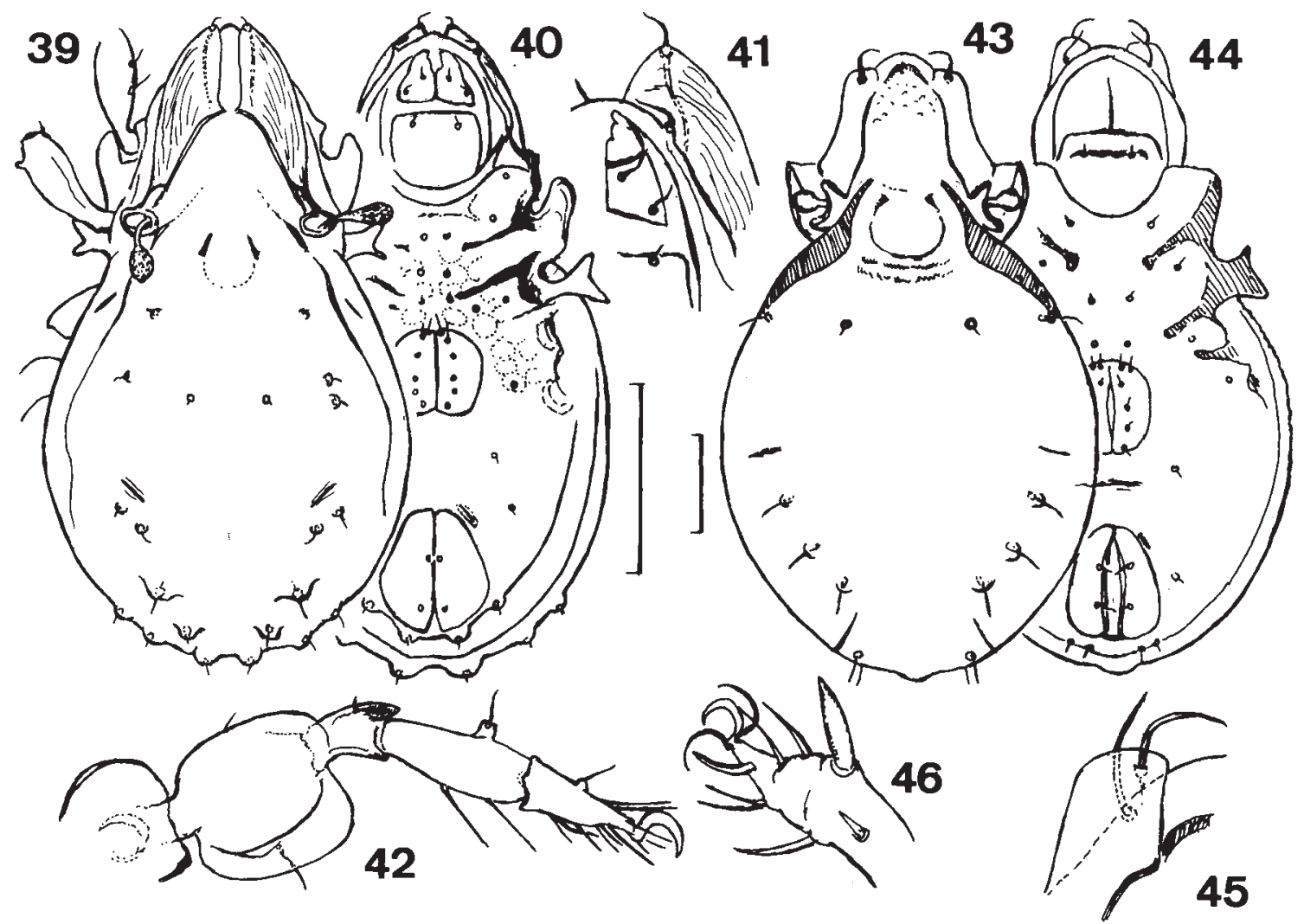

Figs. 39-46.- Scutoverticosus insperatus n. sp.: 39) aspecto dorsal; 40) aspecto ventral; 41) detalle del rostro y la cúspide lamelar, por el lado ventral; 42) pata IV derecha. Ethiovertex elisae n. sp.: 43) aspecto dorsal; 44) aspecto ventral; 45) detalle de la cúspide lamelar izquierda y rostro; 46) tarso III. Escala: $100 \mu \mathrm{m}$.

Figs. 39-46. - Scutoverticosus insperatus n. sp.: 39) dorsal view; 40) ventral view; 41) detail of rostrum and lamellar cusp, ventral view; 42) right leg IV. Ethiovertex elisae n. sp.: 43) dorsal view; 44) ventral view; 45) detail of the left lamellar cusp and the rostrum; 46) tarsus III. Scale: $100 \mu \mathrm{m}$.

Discusión: Sólo se conoce otra especie de este género: S. arcanus Kok, 1968, de Sudáfrica. La especie sudafricana carece de setas le, las ro están barbuladas unilateralmente, las setas $p s$ no se insertan en tubérculos ni tampoco las $a d 1$ y $a d 2$, los apo sj se unen entre si y con los apo 3.

Derivatio nominis: insperatus significa inesperado.

Ethiovertex elisae n. sp. (figs. 43-46)

Holotipo: Un ejemplar de F-5 (MNCN 20.02/9015)

Dimensiones: 605-660 x 374-418. Rostro con visera ampliamente redondeada, en visión ventral se observa a cada lado la seta $r o$, fina, áspera y fuertemente incurvada hacia dentro. Lamelas laterales, sin translamela; cúspides redondeadas por fuera y con un ángulo poco marcado hacia dentro; le parecida a la $r o$; las lamelas llegan por detrás al botridio, que se abre lateralmente; in ausente; ss con cabeza casi esférica y tallo corto. Tutorio desarrollado con punta libre, pedotectos muy grandes. Superficie prodorsal lisa. Borde anterior del notogáster poco preciso; lentícula bien desarrollada. $\mathrm{N}$ : 7, finas, lisas y rectas; fisuras muy patentes. Hipostoma con barra transversal; apodemas cortos y poco desarrollados. Setas coxisternales muy pequeñas, su fórmula es dudosa, parece ser 2-1-2-2. G: 6, dos en el borde anterior. Ag: 1; An: 2 y Ad: 3, 
todas finas, lisas y cortas; $a d 1$ y ad2 postanales; iad paralela al borde anal. Superficie ventral con escultura poco marcada de tuberculitos irregulares. Tarsos tridáctilos heterodáctilos, todos presentan setas convertidas en espinas gruesas y fuertes, una dorsal y otras ventrales.

Discusión: Hasta ahora sólo se conocía la especie tipo: E. macrosetosus Mahunka, 1982, de Etiopía. Según Mahunka (1982) E. macrosetosus tiene talla menor (464-516 x 277-318), borde anterior del notogáster ondulado, setas dorsales pilosas en el lado externo y ss con cabeza mucho más alargada.

Derivatio nominis: Esta especie ha sido dedicada a Elisa Mora, esposa del primero de los autores.

\section{Zygoribatula incomperta $n$. sp. (figs. 47-48)}

Holotipo: Un ejemplar de F-76 (MNCN 20.02/9016)

Dimensiones: 462-520 (495,5) x 300-352 $(330,5)$. Rostro angulado pero no picudo; ro de inserción ligeramente dorsal; lamelas muy estrechas y poco marcadas, sin cúspides, translamela apenas perceptible; le gruesa y áspera; in también gruesas con cortas bárbulas en el extremo. Botridio lateral, parcialmente cubierto por el borde del notogáster; ss con tallo fino y corto y cabeza subesférica; ex finas y rectas. Notogáster subcircular, borde anterior recto; superficie lisa; N: 13 , de ellos $c 1$ sólo un poco más corto que los restantes, todos ásperos y bastante gruesos; setas ps 1 y ps 2 más cortas y finas; áreas porosas redondeadas y grandes; im se aprecia bien, las restantes con dificultad. Superficie coxisternal con escultura poco marcada de grandes alveolos irregulares, setas muy cortas y finas, de fórmula normal. Sólo apo 1 y apo sj están bien desarrollados, apo 4 falta. Orificios genital y anal bien separados; G: 4, cortas y finas; Ag: 1, An: 2 y Ad: 3 (adl postanal, ad 2 paraanal y $a d 3$ preanal), iad preanal. Patas tridáctilas heterodáctilas.

Discusión: La conformación de lamelas y translamela, borde anterior del notogáster y tipo de setas separa bien esta especie de las restantes, muy numerosas, de este género. A la que más recuerda es a Z. arcuata Hammer, 1977, del Pakistán occidental, pero esta especie presenta translamela bien delimitada, setas gastronóticas más cortas y sensilo menos globuloso.

Derivatio nominis: Incompertus en latín quiere decir: no descubierto, no averiguado.

\section{Género Fuerteventuria n. g.}

Familia Protoribatidae. Sensilo formado por tallo corto y cabeza subesférica; surco disyugal ausente; $\mathrm{N}$ : 10; cuatro pares de áreas porosas pequeñas; ausencia de pteromorfos; G: 3 ; sin quitinizaciones salientes dirigidas hacia delante en los acetábulos. Se parece a Paraphauloppia Hammer, pero este género es tridáctilo y existe, a cada lado, una iniciación de surco disyugal.

Especie tipo: Fuerteventuria mirabilis n. sp.

Fuerteventuria mirabilis n. sp. (figs. 49-50)

Holotipo: Un ejemplar de F-31 (MNCN 20.02/9017)

Dimensiones: $303-320(313,72)$ x 133-155 $(146,14)$. Cuerpo alargado y estrecho, coloración amarillenta. Rostro entero ligeramente acuminado; ro insertas lateralmente sobre pequeños tubérculos. Lamelas muy estrechas y laterales, sin cúspides libres; le muy finas y lisas, llegan hasta el extremo del rostro. Botridios muy laterales, abiertos hacia fuera; in muy finas y más cortas que las anteriores, de inserción muy adelantada, a mitad de la distancia entre el botridio y la inserción de $l e ; s s$ con tallo corto y fino y cabeza globulosa, subesférica y casi lisa. No existe surco disyugal, ni siquiera un residuo a cada lado. Cutícula lisa; cuatro pares de áreas porosas circulares y pequeñas. N: 10, finas y poco visibles, lisas y bastante largas. Fisuras en sus emplazamientos habituales, im muy notorias. Sólo apo 1 completo, apo 3 muy corto y apo 4 ausente. Setas coxisternales muy cortas, de fórmula 3-1-3-3. Orificios genital y anal muy separados; G: 3, muy cortas (existe cierta variabilidad, algunos individuos presentan unilateralmente G: 2 y G: 4). An: 2; 

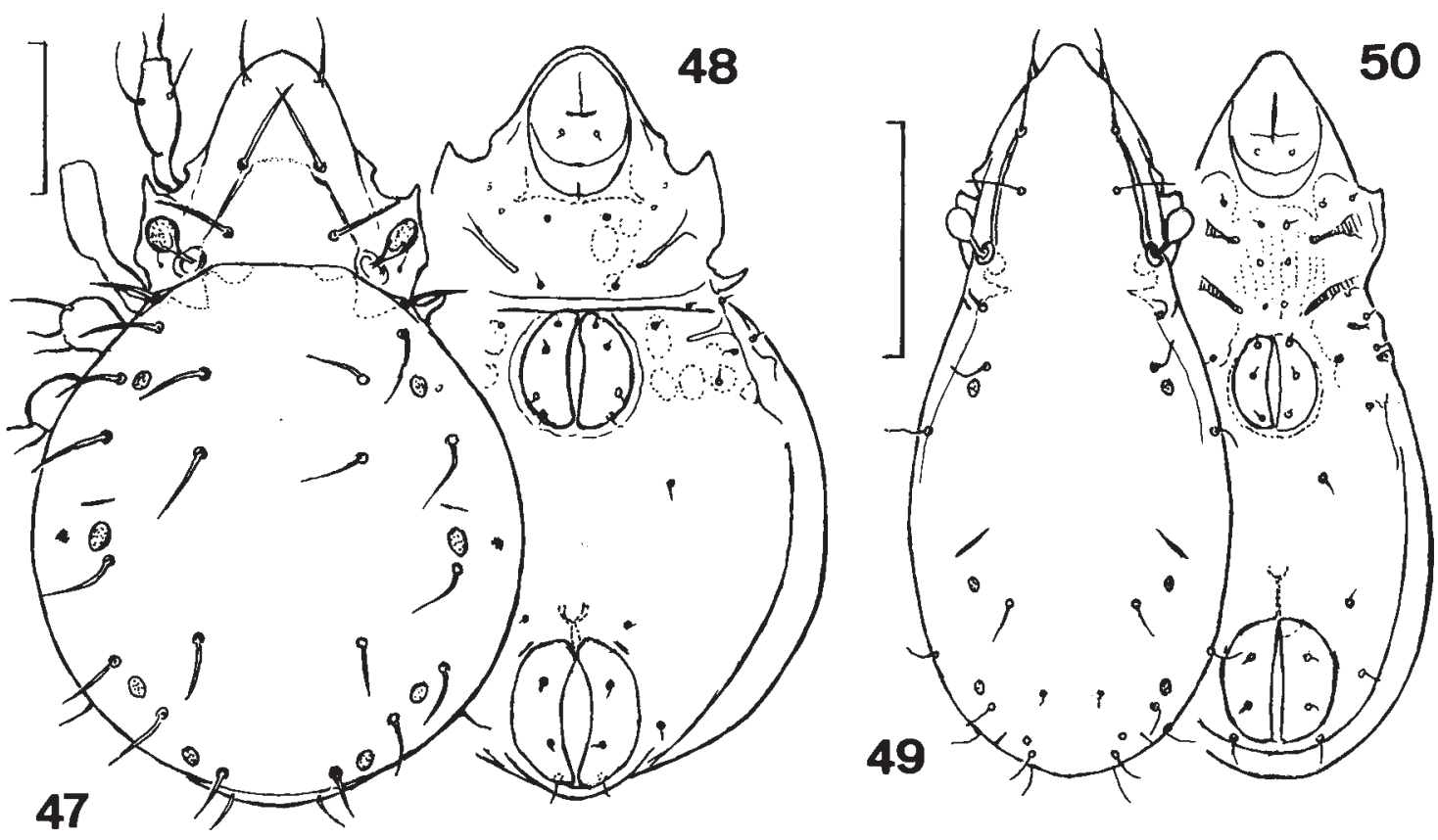

Figs. 47-50.- Zygoribatula incomperta n. sp.: 47) aspecto dorsal; 48) aspecto ventral. Fuerteventuria mirabilis n. g. et n. sp.: 49) aspecto dorsal; 50) aspecto ventral. Escala: $100 \mu \mathrm{m}$.

Figs. 47-50.- Zygoribatula incomperta n. sp.: 47) dorsal view; 48) ventral view. Fuerteventuria mirabilis n. g. et n. sp.: 49) dorsal view; 50) ventral view. Scale: $100 \mu \mathrm{m}$.

Ag: 1 y Ad: 3 (adl postanal y ad3 claramente preanal); iad paralela al borde anal. Tarsos monodáctilos.

Discusión: Esta especie recuerda a Turcibates porosus Ayyildiz \& Luxton, 1989, pero ésta presenta $s s$ mazudos y barbulados, G: 4 y sáculos en vez de áreas porosas.

\section{Lauritzenia (Incabates) depilis $\mathrm{n} . \mathrm{sp}$. (figs. 51-52)}

Holotipo: Un ejemplar de F-74 (MNCN 20.02/9018)

Dimensiones: 320-330 x 200-210. Rostro redondeado; ro bastante largas y barbuladas en su parte externa, insertas muy lateralmente, cerca del extremo del tutorio. Lamelas de situación muy lateral, largas, sin cúspides; le insertas en el extremo, curvas y barbuladas. Botridios de abertura lateral, parcialmente cubiertos por el borde del notogáster; ss con tallo largo y fino, angulado hacia atrás, que se ensancha paulatinamente en una cabeza fusiforme, terminada en punta y provista de bárbulas; in más cortas que las le, más finas y no barbuladas. Superficie prodorsal lisa. Tutorio largo y puntiagudo, más corto que la lamela. Notogáster ovalado, borde anterior ligeramente trilobulado. Pteromorfos triangulares provistos de charnela. N: 10, reducidas al alveolo, que incluso cuesta trabajo visualizar. Cuatro pares de sáculos provistos de un largo tubo, sobre todo $\operatorname{los} S 2$. Fórmula coxisternal normal, setas $2 \mathrm{~A}$ y $3 \mathrm{~A}$ largas, las restantes cortas pero no virtuales. Orificios genital y anal muy separados; G: 4 (reducidas a los alveolos), An:2, Ad:3 y Ag:1, todas virtuales; iad paralela al borde anal; arista circumpédica corta. Patas tridáctilas fuertemente heterodáctilas. 


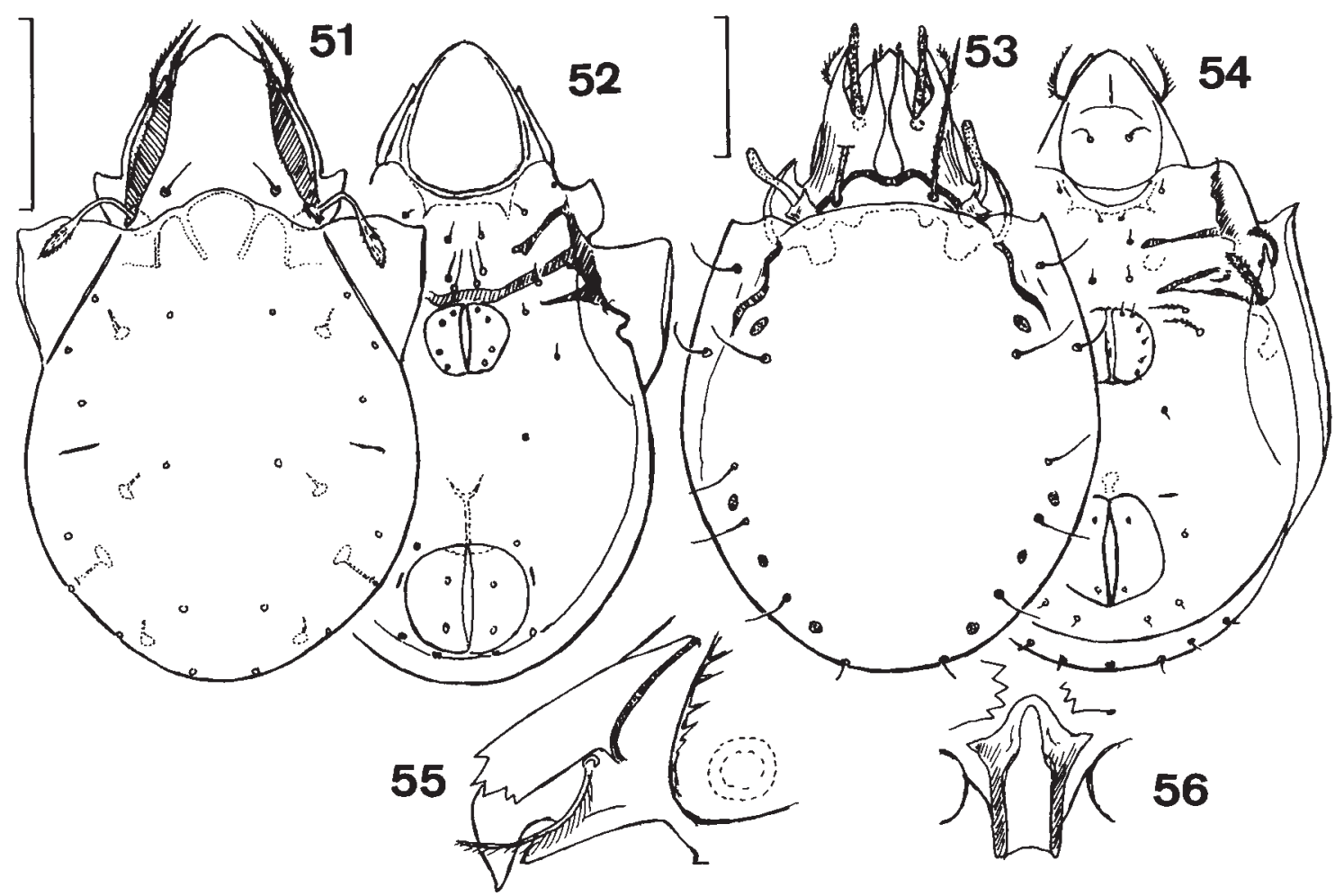

Figs. 51-56. - Lauritzenia (Incabates) depilis n. sp.: 51) aspecto dorsal; 52) aspecto ventral. Oribatella mahani n. sp.: 53) aspecto dorsal; 54) aspecto ventral; 55) tutorio y seta lamelar, aspecto lateral; 56) capuchón rostral, aspecto frontal. Escala: $100 \mu \mathrm{m}$.

Figs. 51-56.- Lauritzenia (Incabates) depilis n. sp.: 51) dorsal view; 52) ventral view. Oribatella mahani n. sp.: 53) dorsal view; 54) ventral view; 55) tutorium and lamellar seta, lateral view; 56) rostral hood, frontal view. Scale: $100 \mu \mathrm{m}$.

Discusión: Esta especie pertenece al grupo con reducción de setas gastronóticas. Se parece a $L$. (I.) nuda Hammer, 1961 pero sus ss son relativamente cortos y con cabeza mazuda, y a L.(I.) angusta Hammer, 1967 cuyos ss tienen cabeza discoidal. latín.

Derivatio nominis: depilis significa lampiño, en

\section{Eupelops claviger fuerteventurae n. subsp.}

Holotipo: Un ejemplar de F-31 (MNCN 20.02/9019)

Dimensiones: 704-748 (731,5) x 528-572 $(555,5)$. Los ejemplares de Fuerteventura presentan los caracteres de la forma nominada, especialmente los descritos y dibujados por Mahunka (1992) a excepción de: 1) Talla, los ejemplares italianos miden $650 \times 470 \mu \mathrm{m}$, por tanto menores que los canarios, y 2) Longitud de las setas gastronóticas que es considerablemente mayor en la subespecie de Fuerteventura.

\section{Oribatella mahani n. sp. (figs. 53-56)}

Holotipo: Un ejemplar de F-27 (MNCN 20.02/9020)

Dimensiones: 427-446 $(434,00)$ x 267-303 $(282,75)$. Rostro acuminado pero no puntiagudo en visión dorsal. Setas ro largas y barbuladas en su borde externo. Lamelas amplias, estriadas en su parte externa, casi en contacto en su parte media; dientes cuspidales largos, espacio cuspidal ancho, con el fondo agudo, donde se inserta le, gruesa y cubierta de bárbulas cortas. Espacio 
interlamelar ensanchado hacia atrás, existe translamela pero no mucrón. Setas in largas, llegan al extremo de las cúspides, y barbuladas; $s s$ en maza alargada de extremo redondeado; tutorio provisto de 4-5 dientes en el extremo, sin dientes en el borde superior; cuatro pares de áreas porosas bastante grandes; N: 10, las setas de los siete pares dorsales bastante largas, sobre todo las anteriores, los tres pares posteriores, insertos en la cara ventral, son más cortos. Apo sj y apo 3 se unen delante del orificio genital; falta apo 4 ; setas coxisternales de fórmula banal, $1 a, 1 b, 2 a$ y $3 b$ cortas, finas y lisas; $1 c$, fina y larga; $4 a$ y $4 b$ más gruesas y barbuladas; $3 c$ y $4 c$ muy gruesas y barbuladas, sobre todo esta última,pero no llega al apodema 2. G: 6, los dos delanteros, en el borde, son los más largos. Ag: 1; An: 2 y Ad: 3, todos finos y lisos; adl y ad2 postanales; iad a la altura del borde anterior del orificio anal,bastante separada y en posición transversal. Area porosa postanal muy pequeña. Capuchón rostral sin dientes, con un saliente romo a cada lado. Patas tridáctilas heterodáctilas.

Discusión: No conocemos ninguna especie de Oribatella tridáctila con translamela y sin mucrón, con seta $4 c$ pilosa y más gruesa y larga que las restantes, pero que no llega al apo 2, tutorio sin dientes en el borde superior y fisura iad alejada del borde anal y de situación transversal.

Derivatio nominis: De Mahan, mítico guerrero prehispánico de la isla, dotado de gran valentía, que media 22 pies de longitud y que, según la leyenda, se encuentra enterrado en la montaña sagrada de Cardones.

\section{COMENTARIOS SOBRE ALGUNAS ESPECIES.}

1) Austrocarabodes arrogans Pérez-Iñigo, 1967: Los ejemplares de Fuerteventura son semejantes a los del centro de la península Ibérica, aunque algo mayores y en algunos ejemplares el prodorso presenta una escultura poco marcada de grandes fosetas irregulares.

2) Scapheremaeus tricarinatus Sitnikova, 1975: Sitnikova (1975) no indica el número de uñas de los tarsos, los ejemplares canarios son monodáctilos, en cambio los ejemplares de El Pardo citados por Mínguez (1981) son tridáctilos; no obstante su parecido los ejemplares de Fuerteventura y los de El Pardo pertenecen a especies diferentes, sin poder precisar cual es el verdadero tricarinatus.

3) Ramusella (Rectoppia) strinatii Mahunka, 1980: Los ejemplares estudiados coinciden con los datos de Mahunka (1980) salvo que son de talla algo menor y que el número de ramas de los sensilos es de 12-13 en los ejemplares nortefricanos y de 8-12 en los canarios.

4) Pseudoamerioppia cf. paraguayensis Balogh \& Mahunka, 1981: El hallazgo de un solo ejemplar impide su determinación segura, pero tanto sus dimensiones (267 x 150) como los caracteres apreciables (setas ro, le y $\mathrm{N}$, ausencia de in y caracteres del ss) coinciden con los datos de Balogh y Mahunka (1981).

5) Licneremaeus similis Pérez-Iñigo Jr., 1989: La ornamentación del notogáster y, sobre todo, de la placa ventral coincide con la propia de L similis, sin embargo las setas gastronóticas son algo más largas, más parecidas a las de L. embeyisztini Mahunka, 1980, de Túnez.

6) Zetomotrichus lacrimans Grandjean, 1934: Los ejemplares de Fuerteventura son ligeramente mayores de lo que indica Grandjean (1934) en los ejemplares de Argelia; hemos comprobado cierta variabilidad en el contorno rostral, que en la mayor parte de los individuos coincide con la forma indicada por Grandjean, pero en algunos la parte central muestra ondulaciones e, incluso, 3-4 pequeños dientes.

7) Zygoribatula lenticulata Mínguez \& Subías, 1986: Los dos ejemplares de Fuerteventura han sido comparados con ejemplares de las islas Columbretes, amablemente cedidos por el Prof. Subías, y se ha llegado a la conclusión de que pertenecen a la misma especie, si bien los ejemplares canarios son un poco mayores, sus setas gastronóticas ligeramente más cortas y con mayor quitinización del notogáster, observándose un retículo, poco señalado, que no se visualiza en la forma mediterránea. Esta especie ha sido hallada también en Pina de Ebro, en la región de Monegros (Gil y Arillo, 1991; Pérez-Iñigo, 1996) y en la costa de Cataluña (Subías, com. pers.). 


\section{Referencias}

BAlogh, J. \& MAhunKa, S., 1981. New data to the knowledge of the oribatid fauna of the Neogaea, VI. (Acari). Acta Zool. Hung., 27(1-2): 49-102.

Grandiean, F., 1934. Oribates de l'Afrique du Nord (2e série). Bull. Soc. Hist. Nat. Afr. Nord, 25: 235-252.

Gil, J. \& ArILlo, A., 1991. Oribátidos de Pina de Ebro (Zaragoza) (Acari, Oribatida). Bol. Asoc. esp. Entomol., 15: 140.

MahunKa, S., 1980. Neue und interessante Milben aus dem Genfer Museum XLII. Erster Beitrag zur Kenntnis der Oribatiden-Fauna der Höhlen Marokkos (Acari: Oribatida). Rev. suisse Zool., 87(3): 797-805.

MahunKa, S., 1982. Oribatids from the Eastern part of the Ethiopian region (Acari). I. Acta Zool. Hung., 28(3-4): 293-336.

MahunKA, S., 1992. "Pelops" and "Oribates" species in the Berlese-collection (Acari). Acta Zool. Hung., 38(3-4): 213-260.

MíngueZ, M.E., 1981. Estudio taxocenótico de los oribátidos (Acarida, Oribatida) de El Pardo. Edit. Univers. Complutense de Madrid, 281 págs.

PÉREZ-Í̃̃̃IGO, C., 1976. Acaros oribátidos de la isla de Tenerife (Acari, Oribatei). II Contribución. Eos, (1975) 51: 85-141.
PÉREZ-ÍÑIGO, C., 1986. Contribución al conocimiento de los oribátidos (Acari, Oribatei) de La Gomera (Islas Canarias). Eos, 62: 187-208.

PÉREZ-Í̃̃IGO, C., 1996. Oribátidos (Acari, Oribatei) hallados sobre plantas en la región de Los Monegros (Aragón, España). Misc. Zool., (1995) 18: 41-46.

Shtanchaeva, U.Ia., 1986. A new species of oribatid mite of the family Passalozetidae from Daghestan. Zool. Zh., 65(9): 1423-1424 (en ruso, resumen en inglés).

Sitnikova, L.G., $1975 . \quad$ Semeistvo Cymbaeremaeidae, En: M.S. Guiliarov y D.A. Krivolutzki (Eds.): Claves para la determinación de ácaros edáficos, Nauka, Moscú, 491 págs. (en ruso). 